\title{
Stringy Hodge numbers and $p$-adic Hodge theory
}

\author{
Tetsushi Ito
}

\begin{abstract}
The aim of this paper is to give an application of $p$-adic Hodge theory to stringy Hodge numbers introduced by $\mathrm{V}$. Batyrev for a mathematical formulation of mirror symmetry. Since the stringy Hodge numbers of an algebraic variety are defined by choosing a resolution of singularities, the well-definedness is not clear from the definition. We give a proof of the well-definedness by using arithmetic techniques such as $p$-adic integration and $p$-adic Hodge theory. Note that another proof of the well-definedness was obtained by V. Batyrev himself by motivic integration.
\end{abstract}

\section{Introduction}

Let $X$ be an irreducible normal algebraic variety over $\mathbb{C}$ with at worst log-terminal singularities. Let $\rho: Y \rightarrow X$ be a resolution of singularities such that the exceptional divisor $\operatorname{Exc}(\rho)$ is a normal crossing divisor whose irreducible components $D_{1}, \ldots, D_{r}$ are smooth. Let $K_{Y}=\rho^{*} K_{X}+\sum_{i=1}^{r} a_{i} D_{i}$ with $a_{i} \in \mathbb{Q}, a_{i}>-1, I:=\{1, \ldots, r\}, D_{J}^{\circ}:=\left(\bigcap_{j \in J} D_{j}\right) \backslash\left(\bigcup_{j \in I \backslash J} D_{j}\right)$ for a nonempty subset $J \subset I$, and $D_{\emptyset}^{\circ}:=Y \backslash \operatorname{Exc}(\rho)$. We define the stringy E-function $E_{\mathrm{st}}(X ; u, v)$ of $X$ by the formula

$$
E_{\mathrm{st}}(X ; u, v):=\sum_{J \subset I} E\left(D_{J}^{\circ} ; u, v\right) \prod_{j \in J} \frac{u v-1}{(u v)^{a_{j}+1}-1},
$$

where $E\left(D_{J}^{\circ} ; u, v\right):=\sum_{k}(-1)^{k} \sum_{i, j} h^{i, j}\left(\operatorname{Gr}_{i+j}^{W} H_{\mathrm{c}}^{k}\left(D_{J}^{\circ}, \mathbb{Q}\right)\right) u^{i} v^{j}$ is the generating function of the Hodge numbers of $D_{J}^{\circ}$ (for details, see $\S 2$ ). The aim of this paper is to give an alternative proof of the following theorem by using arithmetic techniques such as $p$-adic integration and $p$-adic Hodge theory.

Theorem 1.1 [Bat98, Theorem 3.4]. The stringy E-function $E_{\mathrm{st}}(X ; u, v)$ defined as above is independent of the choice of a resolution of singularities $\rho: Y \rightarrow X$.

Assume that $E_{\mathrm{st}}(X ; u, v)$ is a polynomial in $u, v$. We define the stringy Hodge numbers $h_{\mathrm{st}}^{i, j}(X)$ of $X$ by the formula

$$
E_{\mathrm{st}}(X ; u, v)=\sum_{i, j}(-1)^{i+j} h_{\mathrm{st}}^{i, j}(X) u^{i} v^{j} .
$$

Therefore, by Theorem 1.1, we establish the well-definedness of stringy Hodge numbers (also see [Bat98]).

Here we briefly recall a motivation of stringy Hodge numbers. A mathematical formulation of mirror symmetry predicts a symmetry between Hodge numbers of mirror varieties [Bat98, Mor99]. However, some examples discovered by physicists show that the mirror of a smooth variety is

Received 2 December 2002, accepted in final form 31 July 2003, published online 15 October 2004. 2000 Mathematics Subject Classification 11R42, 11S80 (primary), 14E05 (secondary).

Keywords: stringy Hodge numbers, $p$-adic integration, Galois representation, $p$-adic Hodge theory.

This journal is (C) Foundation Compositio Mathematica 2004. 


\section{T. ITо}

not necessarily smooth. In some cases, usual Hodge theory does not work well. To overcome this difficulty, Batyrev introduced stringy Hodge numbers as above [Bat98]. Note that, for proper smooth varieties, stringy Hodge numbers coincide with usual Hodge numbers (Corollary 2.7). Today, several examples of stringy Hodge numbers are computed from the viewpoint of mirror symmetry [BB96, BD96, BM01].

There is an interesting history about the proofs of Theorem 1.1. First of all, Batyrev proved that birational Calabi-Yau manifolds have equal Betti numbers by using arithmetic techniques such as $p$-adic integration and the Weil conjecture [Bat99]. Batyrev's method was generalized to birational smooth minimal models by Wang [Wan98]. The author obtained the equality of Hodge numbers by using $p$-adic Hodge theory [It01, It03]. Wang informed the author that he also obtained the same result independently [Wan2a, Wan2b]. On the other hand, in order to generalize Batyrev's work on Betti numbers to Hodge numbers, M. Kontsevich (in a lecture at Orsay on 7 December 1995) and Denef and Loeser [DL99] developed the theory of motivic integration, which is a geometric analogue of $p$-adic integration. Then, Batyrev introduced stringy Hodge numbers and proved Theorem 1.1 by using motivic integration [Bat98]. In this paper, we give an alternative proof of Theorem 1.1 by using arithmetic techniques such as $p$-adic integration and $p$-adic Hodge theory. In some sense, this paper goes back to Batyrev's original arithmetic approach to Theorem 1.1 by using $p$-adic Hodge theory.

It is worth mentioning that Theorem 1.1 has nontrivial applications to birational geometry. Firstly, for an algebraic variety $X$ over $\mathbb{C}$ with a crepant resolution $\rho: Y \rightarrow X$, the Hodge numbers of $Y$ are independent of the choice of crepant resolution $\rho: Y \rightarrow X$ (Corollary 2.8). This is an important fact in the study of McKay correspondences in higher dimensions [BD96]. Secondly, birational smooth minimal models (e.g. Calabi-Yau manifolds) have equal Hodge numbers (Corollary 2.9). Note that, in dimension $\leqslant 3$, this can also be proved by the minimal model program [KMM85, Kaw88, Kol89]. Recently, a new proof valid in any dimension was given by the weak factorization theorem of birational maps [AKMW02, Vey01, Vey03].

This work is a continuation of the author's previous works [It01, It03]. Here we note the new ingredients of this paper. Basically, the main ideas are the same as before. However, to treat stringy Hodge numbers rather than usual Hodge numbers, we calculate some $p$-adic integration explicitly (Proposition 3.4). Furthermore, to treat combinations of cohomology groups of open varieties, we generalize arithmetic techniques to open varieties by a method of Deligne [Del71a, Del71b] and work on the level of a Grothendieck group of Galois representations rather than individual cohomology groups (see $\S 5$ ).

\section{Stringy Hodge numbers}

In this section, we recall the definition of stringy $E$-functions and stringy Hodge numbers as in [Bat98].

For an algebraic variety $X$ over $\mathbb{C}$, the cohomology groups with compact support $H_{\mathrm{c}}^{k}(X, \mathbb{Q})$ have canonical mixed Hodge structures by Deligne [Del71b, Del74b]. Let $W$ be the weight filtration on $H_{\mathrm{c}}^{k}(X, \mathbb{Q})$. Each graded quotient $\operatorname{Gr}_{l}^{W} H_{\mathrm{c}}^{k}(X, \mathbb{Q})$ has a pure Hodge structure of weight $l$. Let $h^{i, j}\left(\mathrm{Gr}_{i+j}^{W} H_{\mathrm{c}}^{k}(X, \mathbb{Q})\right)$ be the dimension of the $(i, j)$ th Hodge component of $\operatorname{Gr}_{i+j}^{W} H_{\mathrm{c}}^{k}(X, \mathbb{Q})$ for each $i, j$.

Definition 2.1. We define the E-function of $X$ as follows:

$$
E(X ; u, v):=\sum_{k}(-1)^{k} \sum_{i, j} h^{i, j}\left(\operatorname{Gr}_{i+j}^{W} H_{\mathrm{c}}^{k}(X, \mathbb{Q})\right) u^{i} v^{j} .
$$




\section{STRINGY HodGe NUMBERS}

For a proper smooth variety $X$ over $\mathbb{C}$,

$$
E(X ; u, v)=\sum_{i, j}(-1)^{i+j} h^{i, j}(X) u^{i} v^{j},
$$

where $h^{i, j}(X):=\operatorname{dim} H^{j}\left(X, \Omega_{X}^{i}\right)$ are the Hodge numbers of $X$ as usual.

Remark 2.2. The $E$-function satisfies the following properties:

1) for $Z \subset X$, we have $E(X ; u, v)=E(X \backslash Z ; u, v)+E(Z ; u, v)$;

2) for $X, Y$, we have $E(X \times Y ; u, v)=E(X ; u, v) \cdot E(Y ; u, v)$.

These two properties imply that it is natural to consider the $E$-function as a ring homomorphism from a Grothendieck group of algebraic varieties over $\mathbb{C}$ to $\mathbb{Z}[u, v]$ [DL98, DL99, DL01].

Let $X$ be an irreducible normal algebraic variety over $\mathbb{C}$, and $\rho: Y \rightarrow X$ be a resolution of singularities such that the exceptional divisor $\operatorname{Exc}(\rho)$ is a simple normal crossing divisor (SNCD). Recall that a normal crossing divisor (NCD) is simple if its irreducible components are smooth. Let the irreducible components of $\operatorname{Exc}(\rho)$ be $D_{1}, \ldots, D_{r}$.

Definition 2.3 [Bat98, Definition 2.2]. The variety $X$ is said to have at worst log-terminal singularities if the following conditions are satisfied:

1) the canonical divisor $K_{X}$ is a $\mathbb{Q}$-Cartier divisor (i.e. $X$ is $\mathbb{Q}$-Gorenstein);

2) we have

$$
K_{Y}=\rho^{*} K_{X}+\sum_{i=1}^{r} a_{i} D_{i} \quad\left(a_{i} \in \mathbb{Q}\right),
$$

with $a_{i}>-1$ (note that this condition is independent of the choice of resolution $\rho: Y \rightarrow X$ ).

Let $X, Y$ be as above and $X$ have at worst log-terminal singularities. Let $I:=\{1, \ldots, r\}$. For any subset $J \subset I$, we set

$$
D_{J}:=\left\{\begin{array}{ll}
\bigcap_{j \in J} D_{j} & J \neq \emptyset, \\
Y & J=\emptyset,
\end{array} \quad D_{J}^{\circ}:=D_{J} \backslash \bigcup_{j \in I \backslash J} D_{j} .\right.
$$

Definition 2.4 [Bat98, Definition 3.1]. We define the stringy E-function of $X$ as follows:

$$
E_{\mathrm{st}}(X ; u, v):=\sum_{J \subset I} E\left(D_{J}^{\circ} ; u, v\right) \prod_{j \in J} \frac{u v-1}{(u v)^{a_{j}+1}-1},
$$

where $E\left(D_{J}^{\circ} ; u, v\right)$ is the $E$-function of a smooth variety $D_{J}^{\circ}$ defined at the beginning of this section.

Remark 2.5. Since $X$ has at worst log-terminal singularities, $a_{i}+1>0$ and hence the denominator of $E_{\mathrm{st}}(X ; u, v)$ does not vanish (also see Remark 3.7$)$. In general, $E_{\mathrm{st}}(X ; u, v)$ is an element of $\mathbb{Q}\left(u^{1 / d}, v^{1 / d}\right) \cap \mathbb{Z}\left[\left[u^{1 / d}, v^{1 / d}\right]\right]$, where $d$ is the least common multiplier of the denominators of $a_{i}$.

Definition 2.6. Assume that $E_{\mathrm{st}}(X ; u, v)$ is a polynomial in $u, v$. Then we define the stringy Hodge numbers $h_{\mathrm{st}}^{i, j}(X)$ of $X$ by the formula

$$
E_{\mathrm{st}}(X ; u, v)=\sum_{i, j}(-1)^{i+j} h_{\mathrm{st}}^{i, j}(X) u^{i} v^{j} .
$$

Theorem 1.1 claims that $E_{\mathrm{st}}(X ; u, v)$ is independent of the choice of resolution $\rho: Y \rightarrow X$. Once we know the well-definedness, we can prove some fundamental properties of $E_{\mathrm{st}}(X ; u, v)$ as in [Bat98].

Here we list some immediate corollaries of Theorem 1.1. 
Corollary 2.7 [Bat98, Corollary 3.6]. If $X$ is smooth, then we have $E_{\mathrm{st}}(X ; u, v)=E(X ; u, v)$.

Proof. This is clear because the identity map id :X $\rightarrow X$ is a resolution of singularities.

Corollary 2.8 [Bat98, Theorem 3.12]. Let $X$ be a projective algebraic variety over $\mathbb{C}$ which has a crepant resolution $\rho: Y \rightarrow X$ (i.e. $\rho: Y \rightarrow X$ is a resolution of singularities with $\rho^{*} K_{X}=K_{Y}$ ). Then the stringy Hodge numbers of $X$ are equal to the Hodge numbers of $Y$ :

$$
h_{\mathrm{st}}^{i, j}(X)=h^{i, j}(Y) \text { for all } i, j .
$$

In particular, the Hodge numbers of $Y$ are independent of the choice of crepant resolution $\rho: Y \rightarrow X$. Moreover, we can compute the Hodge numbers of a crepant resolution of $X$ via any resolution of singularities which is not necessarily crepant.

Proof. This is clear because we have $E_{\mathrm{st}}(X ; u, v)=E(Y ; u, v)$ by Definition 2.4.

Corollary 2.9 [Bat99, Bat98, Vey01, Vey03, Wan98, Wan2a, Wan2b, It01, It03]. Let $X, Y$ be projective smooth algebraic varieties over $\mathbb{C}$ whose canonical bundles are NEF (i.e. $X, Y$ are minimal models). Assume that $X, Y$ are birational. Then $X, Y$ have equal Hodge numbers:

$$
h^{i, j}(X)=h^{i, j}(Y) \text { for all } i, j \text {. }
$$

Proof. Let $f: X \rightarrow Y$ be a birational map. Then we can find a projective smooth algebraic variety $Z$ over $\mathbb{C}$ and birational morphisms $g: Z \rightarrow X, h: Z \rightarrow Y$ such that $f \circ g=h$ as birational maps and $g^{*} K_{X}=h^{*} K_{Y}$.

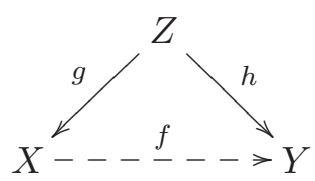

This is a standard fact in birational geometry (for example, see [It03, Proposition 2.1]). We consider $g: Z \rightarrow X$ (respectively $h: Z \rightarrow Y$ ) as a resolution of singularities of $X$ (respectively $Y$ ) and calculate the stringy Hodge numbers of $X$ (respectively $Y$ ). Since $g^{*} K_{X}=h^{*} K_{Y}$, we have

$$
E(X ; u, v)=E_{\mathrm{st}}(X ; u, v)=E_{\mathrm{st}}(Y ; u, v)=E(Y ; u, v) .
$$

Hence we have the equality of the Hodge numbers of $X, Y$.

\section{3. $p$-adic integration}

In this section, we recall Weil's $p$-adic integration developed in [Wei82] which is an important tool to count the number of rational points valued in a finite field.

\subsection{Setup}

Let $p$ be a prime number and $\mathbb{Q}_{p}$ be the field of $p$-adic numbers. Let $F$ be a finite extension of $\mathbb{Q}_{p}$, $R \subset F$ be the ring of integers in $F, m \subset R$ be the maximal ideal of $R$, and $\mathbb{F}_{q}=R / m$ be the residue field of $F$ with $q$ elements, where $q$ is a power of $p$. For an element $x \in F$, we define the $p$-adic absolute value $|x|_{p}$ by

$$
|x|_{p}:= \begin{cases}q^{-v(x)} & x \neq 0, \\ 0 & x=0,\end{cases}
$$

where $v: F^{\times} \rightarrow \mathbb{Z}$ is the normalized discrete valuation of $F$.

Let $\mathfrak{X}$ be a smooth scheme over $R$ of relative dimension $n$. We can compute the number of $\mathbb{F}_{q^{-}}$ rational points $\left|\mathfrak{X}\left(\mathbb{F}_{q}\right)\right|$ by integrating a certain $p$-adic measure on the set of $R$-rational points $\mathfrak{X}(R)$. 


\section{StRingy Hodge NUMBers}

We note that $\mathfrak{X}(R)$ is a compact and totally disconnected topological space with respect to its $p$-adic topology.

\section{$3.2 p$-adic integration of regular $n$-forms}

Let $\omega \in \Gamma\left(\mathfrak{X}, \Omega_{\mathfrak{X} / R}^{n}\right)$ be a regular $n$-form on $\mathfrak{X}$, where $\Omega_{\mathfrak{X} / R}^{n}$ is the relative canonical bundle of $\mathfrak{X} / R$. We shall define the $p$-adic integration of $\omega$ on $\mathfrak{X}(R)$ as follows. Let $s \in \mathfrak{X}(R)$ be an $R$-rational point. Let $U \subset \mathfrak{X}(R)$ be a sufficiently small $p$-adic open neighborhood of $s$ on which there exists a system of local $p$-adic coordinates $\left\{x_{1}, \ldots, x_{n}\right\}$. Then $\left\{x_{1}, \ldots, x_{n}\right\}$ defines a $p$-adic analytic map

$$
x=\left(x_{1}, \ldots, x_{n}\right): U \longrightarrow R^{n},
$$

which is a homeomorphism between $U$ and a $p$-adic open set $V$ of $R^{n}$. By using the above coordinates, $\omega$ is written as

$$
\omega=f(x) d x_{1} \wedge \cdots \wedge d x_{n} .
$$

We consider $f(x)$ as a $p$-adic analytic function on $V$. Then we define the p-adic integration of $\omega$ on $U$ by the equation

$$
\int_{U}|\omega|_{p}:=\int_{V}|f(x)|_{p} d x_{1} \cdots d x_{n}
$$

where $|f(x)|_{p}$ is the $p$-adic absolute value of the value of $f$ at $x \in V$ and $d x_{1} \cdots d x_{n}$ is the Haar measure on $R^{n}$ normalized by the condition

$$
\int_{R^{n}} d x_{1} \cdots d x_{n}=1
$$

By patching them, we get the $p$-adic integration of $\omega$ on $\mathfrak{X}(R)$ :

$$
\int_{\mathfrak{X}(R)}|\omega|_{p} .
$$

\section{$3.3 p$-adic integration of gauge forms}

By definition, a gauge form $\omega$ on $\mathfrak{X}$ is a nowhere vanishing global section $\omega \in \Gamma\left(\mathfrak{X}, \Omega_{\mathfrak{X} / R}^{n}\right)$. The most important property of $p$-adic integration is that the $p$-adic integration of a gauge form computes the number of $\mathbb{F}_{q}$-rational points.

Proposition 3.1 [Wei82, 2.2.5]. Let $\mathfrak{X}$ be a smooth scheme over $R$ of relative dimension $n$ and $\omega$ be a gauge form on $\mathfrak{X}$. Then

$$
\int_{\mathfrak{X}(R)}|\omega|_{p}=\frac{\left|\mathfrak{X}\left(\mathbb{F}_{q}\right)\right|}{q^{n}} .
$$

Proof. Let

$$
\varphi: \mathfrak{X}(R) \longrightarrow \mathfrak{X}\left(\mathbb{F}_{q}\right)
$$

be the reduction map. For $\bar{x} \in \mathfrak{X}\left(\mathbb{F}_{q}\right), \varphi^{-1}(\bar{x})$ is a $p$-adic open set of $\mathfrak{X}(R)$. Therefore, it is enough to show

$$
\int_{\varphi^{-1}(\bar{x})}|\omega|_{p}=\frac{1}{q^{n}}
$$

Let $\left\{x_{1}, \ldots, x_{n}\right\} \subset \mathcal{O}_{\mathfrak{X}, \bar{x}}$ be a regular system of parameters at $\bar{x}$. Then $\left\{x_{1}, \ldots, x_{n}\right\}$ defines a system of local $p$-adic coordinates on $\varphi^{-1}(\bar{x})$ and

$$
x=\left(x_{1}, \ldots, x_{n}\right): \varphi^{-1}(\bar{x}) \longrightarrow m^{n} \subset R^{n}
$$


is a $p$-adic analytic homeomorphism. Let $\omega$ be written as $\omega=f(x) d x_{1} \wedge \cdots \wedge d x_{n}$. Since $\omega$ is a gauge form, $f(x)$ is a $p$-adic unit for all $x \in \varphi^{-1}(\bar{x})$. Therefore $|f(x)|_{p}=1$. Then we have

$$
\int_{\varphi^{-1}(\bar{x})}|\omega|_{p}=\int_{m^{n}} d x_{1} \cdots d x_{n}=\frac{1}{q^{n}},
$$

since $m^{n}$ is an index $q^{n}$ subgroup of $R^{n}$.

\subsection{Computation of some $p$-adic integration}

Since we treat stringy Hodge numbers rather than usual Hodge numbers in this paper, we need to compute some $p$-adic integration slightly more general than Proposition 3.1.

Firstly, we generalize $p$-adic integration to an $r$-pluricanonical form with pole for $r \in \mathbb{Z}, r \geqslant 1$ (see [Wan98]). An $r$-pluricanonical form on $\mathfrak{X}$ is a section of $\left(\Omega_{\mathfrak{X} / R}^{n}\right)^{\otimes r}$ over $\mathfrak{X}$. An $r$-pluricanonical form with pole on $\mathfrak{X}$ is a section of $\left(\Omega_{\mathfrak{U} / R}^{n}\right)^{\otimes r}$ over $\mathfrak{U}$ for some open subscheme $\mathfrak{U} \subset \mathfrak{X}$. Let $\omega$ be an $r$-pluricanonical form with pole on $\mathfrak{X}$. As in the case of a regular $n$-form, locally in $p$-adic topology, $\omega$ is written as

$$
\omega=f(x)\left(d x_{1} \wedge \cdots \wedge d x_{n}\right)^{\otimes r}
$$

for a system of local $p$-adic coordinates $\left\{x_{1}, \ldots, x_{n}\right\}$. Note that $f(x)$ is a $p$-adic analytic function with pole. Then we put

$$
\int_{U}|\omega|_{p}^{1 / r}:=\int_{V}|f(x)|_{p}^{1 / r} d x_{1} \cdots d x_{n}
$$

where $U, V$ are the same as in the case of a regular $n$-form, if the right-hand side converges. If the above integral converges for each open neighborhood, by patching them, we get the p-adic integration of an r-pluricanonical form with pole $\omega$ on $\mathfrak{X}(R)$ :

$$
\int_{\mathfrak{X}(R)}|\omega|_{p}^{1 / r}
$$

Note that, if $\omega$ has no pole, the above integral always converges.

Remark 3.2. For an $r$-pluricanonical form with pole $\omega, \omega^{\otimes s}$ is an $r s$-pluricanonical form with pole. If the $p$-adic integration of $\omega$ converges, then the $p$-adic integration of $\omega^{\otimes s}$ also converges and they are equal:

$$
\int_{\mathfrak{X}(R)}|\omega|_{p}^{1 / r}=\int_{\mathfrak{X}(R)}\left|\omega^{\otimes s}\right|_{p}^{1 / r s} .
$$

Before computing $p$-adic integration, we recall the notion of relative SNCD.

Definition 3.3. Let $f: \mathfrak{X} \rightarrow S$ be a proper smooth morphism of schemes and $\mathfrak{D}=\sum_{i=1}^{r} a_{i} \mathfrak{D}_{i}$ $\left(a_{i} \in \mathbb{Q}, a_{i} \neq 0\right)$ be a $\mathbb{Q}$-divisor on $\mathfrak{X}$. Let $\operatorname{Supp} \mathfrak{D}=\bigcup_{i=1}^{r} \mathfrak{D}_{i}$ be the support of $\mathfrak{D}$. Then, $\mathfrak{D}$ is called a relative $S N C D$ (simple normal crossing divisor) on $\mathfrak{X} / S$ if all $\mathfrak{D}_{i}$ are smooth over $S$ and, for all $x \in \operatorname{Supp} \mathfrak{D}$, the completion of $\operatorname{Supp} \mathfrak{D} \hookrightarrow \mathfrak{X}$ at $x$ is isomorphic to

$$
\operatorname{Spec}\left(\widehat{\mathcal{O}}_{S, f(x)}\left[\left[x_{1}, \ldots, x_{d}\right]\right] /\left(x_{1} \cdots x_{s}\right)\right) \hookrightarrow \operatorname{Spec}\left(\widehat{\mathcal{O}}_{S, f(x)}\left[\left[x_{1}, \ldots, x_{d}\right]\right]\right)
$$

for some $s(1 \leqslant s \leqslant d)$, where $d$ is the relative dimension of $f$.

Note that $\widehat{\mathcal{O}}_{\mathfrak{X}, x}$ is isomorphic to $\widehat{\mathcal{O}}_{S, f(x)}\left[\left[x_{1}, \ldots, x_{d}\right]\right]$ because $f$ is smooth of relative dimension $d$. In this case, for a nonempty subset $J \subset\{1, \ldots, r\}, \bigcap_{j \in J} \mathfrak{D}_{j}$ is smooth of relative dimension $d-|J|$ over $S$. 


\section{STRINGY HodGe NUMBERS}

We shall compute some $p$-adic integration. Let $\mathfrak{X}$ be a smooth scheme over $R$ of relative dimension $n$, and $\omega$ an $r$-pluricanonical form with pole on $\mathfrak{X}$. Assume that

$$
\operatorname{div}(\omega)=\sum_{i=1}^{s} a_{i} \mathfrak{D}_{i}
$$

is a relative $\mathrm{SNCD}$ on $\mathfrak{X} / R$. Let $I:=\{1, \ldots, s\}$. For any subset $J \subset I$, we set

$$
\mathfrak{D}_{J}:=\left\{\begin{array}{ll}
\bigcap_{j \in J} \mathfrak{D}_{j} & J \neq \emptyset, \\
\mathfrak{X} & J=\emptyset,
\end{array} \quad \mathfrak{D}_{J}^{\circ}:=\mathfrak{D}_{J} \backslash \bigcup_{j \in I \backslash J} \mathfrak{D}_{j} .\right.
$$

Proposition 3.4. Let the notation be as above. If $a_{i}>-r$ for all $i \in I$, then the $p$-adic integration of $\omega$ on $\mathfrak{X}(R)$ converges, and we have the following equality:

$$
\int_{\mathfrak{X}(R)}|\omega|_{p}^{1 / r}=\frac{1}{q^{n}} \sum_{J \subset I}\left|\mathfrak{D}_{J}^{\circ}\left(\mathbb{F}_{q}\right)\right| \prod_{j \in J} \frac{q-1}{q^{\left(a_{j} / r\right)+1}-1} .
$$

If $r=1$ and $\omega$ is a gauge form, Proposition 3.4 is nothing but Proposition 3.1.

Proof. The idea of the proof is the same as in Proposition 3.1. Let

$$
\varphi: \mathfrak{X}(R) \longrightarrow \mathfrak{X}\left(\mathbb{F}_{q}\right)
$$

be the reduction map. For $\bar{x} \in \mathfrak{X}\left(\mathbb{F}_{q}\right), \varphi^{-1}(\bar{x})$ is a $p$-adic open set of $\mathfrak{X}(R)$. Therefore, it is enough to show that

$$
\int_{\varphi^{-1}(\bar{x})}|\omega|_{p}^{1 / r}=\frac{1}{q^{n}} \prod_{j \in I \mid \bar{x} \in \mathfrak{D}_{j}\left(\mathbb{F}_{q}\right)} \frac{q-1}{q^{\left(a_{j} / r\right)+1}-1} .
$$

Let $\left\{j_{1}, \ldots, j_{k}\right\}=\left\{j \in I \mid \bar{x} \in \mathfrak{D}_{j}\left(\mathbb{F}_{q}\right)\right\}$. Let $\left\{x_{1}, \ldots, x_{n}\right\} \subset \mathcal{O}_{\mathfrak{X}, \bar{x}}$ be a regular system of parameters at $\bar{x}$ such that $\mathfrak{D}_{j_{i}}$ is defined by $x_{i}=0$ at $\bar{x}$ for all $i=1, \ldots, k$. Then $\left\{x_{1}, \ldots, x_{n}\right\}$ defines a system of local $p$-adic coordinates on $\varphi^{-1}(\bar{x})$ and

$$
x=\left(x_{1}, \ldots, x_{n}\right): \varphi^{-1}(\bar{x}) \longrightarrow m^{n} \subset R^{n}
$$

is a $p$-adic analytic homeomorphism. Here $\omega$ is written as

$$
\omega=f(x) \cdot x_{1}^{a_{j_{1}}} \cdots x_{k}^{a_{j_{k}}}\left(d x_{1} \wedge \cdots \wedge d x_{n}\right)^{\otimes r},
$$

where $f(x)$ is a $p$-adic unit for all $x \in \varphi^{-1}(\bar{x})$. Hence we have

$$
\left|f(x) \cdot x_{1}^{a_{j_{1}}} \cdots x_{k}^{a_{j_{k}}}\right|_{p}^{1 / r}=\left|x_{1}\right|_{p}^{a_{j_{1}} / r} \cdots\left|x_{k}\right|_{p}^{a_{j_{k}} / r},
$$

and

$$
\begin{aligned}
\int_{\varphi^{-1}(\bar{x})}|\omega|_{p}^{1 / r} & =\int_{m^{n}}\left|x_{1}\right|_{p}^{a_{j_{1}} / r} \cdots\left|x_{k}\right|_{p}^{a_{j_{k}} / r} d x_{1} \cdots d x_{n} \\
& =\frac{1}{q^{n-k}}\left(\int_{m^{k}}\left|x_{1}\right|_{p}^{a_{j_{1}} / r} \cdots\left|x_{k}\right|_{p}^{a_{j_{k}} / r} d x_{1} \cdots d x_{k}\right) .
\end{aligned}
$$

Therefore, it is enough to prove the following lemma.

Lemma 3.5. For $k_{1}, \ldots, k_{n} \in \mathbb{Q}, k_{i}>-1$, the $p$-adic integration

$$
\int_{m^{n}}\left|x_{1}\right|_{p}^{k_{1}} \cdots\left|x_{n}\right|_{p}^{k_{n}} d x_{1} \cdots d x_{n}
$$

converges and is equal to

$$
\frac{1}{q^{n}} \prod_{i=1}^{n} \frac{q-1}{q^{k_{i}+1}-1}
$$


Proof. By iterated integration, we have

$$
\int_{m^{n}}\left|x_{1}\right|_{p}^{k_{1}} \cdots\left|x_{n}\right|_{p}^{k_{n}} d x_{1} \cdots d x_{n}=\left(\int_{m}\left|x_{1}\right|_{p}^{k_{1}} d x_{1}\right) \cdots\left(\int_{m}\left|x_{n}\right|_{p}^{k_{n}} d x_{n}\right) .
$$

Therefore, it is enough to prove

$$
\int_{m}|x|_{p}^{k} d x=\frac{1}{q} \cdot \frac{q-1}{q^{k+1}-1}
$$

for $k \in \mathbb{Q}, k>-1$.

We compute the above integration by dividing $m$ as a disjoint union of open subsets as follows:

$$
m=\coprod_{i=1}^{\infty} m^{i} \backslash m^{i+1}
$$

For $x \in m^{i} \backslash m^{i+1},|x|_{p}^{k}=q^{-k i}$. The volume of $m^{i}$ is $q^{-i}$ with respect to the normalized Haar measure on $R$ since $m^{i}$ is an index $q^{i}$ subgroup of $R$. Therefore, we have

$$
\begin{aligned}
\int_{m}|x|_{p}^{k} d x & =\sum_{i=1}^{\infty} q^{-i k} \operatorname{vol}\left(m^{i} \backslash m^{i+1}\right)=\sum_{i=1}^{\infty} q^{-i k}\left(q^{-i}-q^{-(i+1)}\right) \\
& =\left(1-q^{-1}\right) \sum_{i=1}^{\infty}\left(q^{-(k+1)}\right)^{i} .
\end{aligned}
$$

Since $k>-1$, this infinite sum converges to

$$
\left(1-q^{-1}\right) \cdot \frac{q^{-(k+1)}}{1-q^{-(k+1)}}=\frac{1}{q} \cdot \frac{q-1}{q^{k+1}-1} .
$$

Hence we have proved Lemma 3.5, and the proof of Proposition 3.4 is complete.

Remark 3.6. A curious reader may notice the similarity between the expression in Proposition 3.4 and Definition 2.4. This is the starting point of our proof of Theorem 1.1. However, to recover information of the Hodge numbers of an algebraic variety from the number of rational points valued in finite fields, we need some deep arithmetic results as in $\S \S 4$ and 5.

Remark 3.7. As we easily see in the proof of Lemma 3.5, the p-adic integration

$$
\int_{m^{n}}\left|x_{1}\right|_{p}^{k_{1}} \cdots\left|x_{n}\right|_{p}^{k_{n}} d x_{1} \cdots d x_{n}
$$

does not converge if $k_{i} \leqslant-1$ for some $i$. This is the reason why we assume singularities are at worst log-terminal in Theorem 1.1 .

\section{Local Galois representations}

In this section, we recall some results on Galois representations over a $p$-adic field.

\subsection{Setup}

Let $K$ be a number field. Let $\mathfrak{p}$ be a maximal ideal of $\mathcal{O}_{K}$. Let $K_{\mathfrak{p}}$ be a $\mathfrak{p}$-adic completion of $K, \mathcal{O}_{K_{\mathfrak{p}}}$ the ring of integers of $K_{\mathfrak{p}}, \mathbb{F}_{q}=\mathcal{O}_{K} / \mathfrak{p}$ the residue field of $K_{\mathfrak{p}}$ with $q$ elements, and $\bar{K}_{\mathfrak{p}}$ (respectively $\overline{\mathbb{F}}_{q}$ ) an algebraic closure of $K_{\mathfrak{p}}$ (respectively $\mathbb{F}_{q}$ ).

We have an exact sequence

$$
0 \longrightarrow I_{K_{\mathfrak{p}}} \longrightarrow \operatorname{Gal}\left(\bar{K}_{\mathfrak{p}} / K_{\mathfrak{p}}\right) \longrightarrow \operatorname{Gal}\left(\overline{\mathbb{F}}_{q} / \mathbb{F}_{q}\right) \longrightarrow 0,
$$




\section{Stringy Hodge NUmbers}

where $I_{K_{\mathfrak{p}}}$ is called the inertia group at $\mathfrak{p}$. The group $\operatorname{Gal}\left(\overline{\mathbb{F}}_{q} / \mathbb{F}_{q}\right)$ is topologically generated by the $q$ th power Frobenius automorphism $x \mapsto x^{q}$ of $\overline{\mathbb{F}}_{q}$. The inverse of this automorphism is called the geometric Frobenius element at $\mathfrak{p}$ and is denoted by Frob

Let $X$ be a proper smooth variety over $K_{\mathfrak{p}}, l$ be a prime number, and $k$ be an integer. Then the absolute Galois group $\operatorname{Gal}\left(\bar{K}_{\mathfrak{p}} / K_{\mathfrak{p}}\right)$ acts continuously on the $l$-adic étale cohomology group $H_{\text {ét }}^{k}\left(X_{\bar{K}_{\mathfrak{p}}}, \mathbb{Q}_{l}\right)$ of $X_{\bar{K}_{\mathfrak{p}}}=X \otimes_{K_{\mathfrak{p}}} \bar{K}_{\mathfrak{p}}$. In the following, we recall some results on this $\operatorname{Gal}\left(\bar{K}_{\mathfrak{p}} / K_{\mathfrak{p}}\right)$ representation in two cases.

\subsection{The Weil conjecture}

Firstly, we assume that $\mathfrak{p}$ does not divide $l$ and there exists a proper smooth scheme $\mathfrak{X}$ over $\mathcal{O}_{K_{\mathfrak{p}}}$ such that $\mathfrak{X} \otimes \mathcal{O}_{K_{\mathfrak{p}}} K_{\mathfrak{p}}=X$ ( $\mathfrak{X}$ is called a proper smooth model of $X$ over $\mathcal{O}_{K_{\mathfrak{p}}}$ ).

In this case, the action of $I_{K_{\mathfrak{p}}}$ on $H_{\text {êt }}^{k}\left(X_{\bar{K}_{\mathfrak{p}}}, \mathbb{Q}_{l}\right)$ is trivial (i.e. the action of $\operatorname{Gal}\left(\bar{K}_{\mathfrak{p}} / K_{\mathfrak{p}}\right)$ is unramified) by the proper smooth base change theorem on étale cohomology. Therefore, the action of $\operatorname{Gal}\left(\bar{K}_{\mathfrak{p}} / K_{\mathfrak{p}}\right)$ is determined by the action of Frob .

By the Lefschetz trace formula for étale cohomology, we have

$$
\left|\mathfrak{X}\left(\mathbb{F}_{q}\right)\right|=\sum_{k}(-1)^{k} \operatorname{Tr}\left(\text { Frob }_{\mathfrak{p}} ; H_{\text {ét }}^{k}\left(X_{\bar{K}_{\mathfrak{p}}}, \mathbb{Q}_{l}\right)\right) .
$$

Furthermore, the characteristic polynomial $P_{k}(t)=\operatorname{det}\left(1-t \cdot\right.$ Frob $\left._{\mathfrak{p}} ; H_{\text {ét }}^{k}\left(X_{\bar{K}_{\mathfrak{p}}}, \mathbb{Q}_{l}\right)\right)$ has integer coefficients and all complex absolute values of all conjugates of the roots of $P_{k}(t)$ are equal to $q^{-k / 2}$ by the Weil conjecture proved by Deligne [Del74a, Del80].

\section{$4.3 p$-adic Hodge theory}

Secondly, we assume $\mathfrak{p}$ divides $l$. Let $p=l$ in this subsection to avoid confusion. Here no assumption is required for a model of $X$ over $\mathcal{O}_{K_{\mathfrak{p}}}$ (see Remark 4.1). In this case, the action of the inertia group $I_{K_{\mathfrak{p}}}$ on $H_{\text {ét }}^{k}\left(X_{\bar{K}}, \mathbb{Q}_{p}\right)$ is highly nontrivial.

Let $\mathbb{C}_{p}$ be a $\mathfrak{p}$-adic completion of $\bar{K}_{\mathfrak{p}}$ on which $\operatorname{Gal}\left(\bar{K}_{\mathfrak{p}} / K_{\mathfrak{p}}\right)$ acts continuously. We recall the Tate twists. Let $\mathbb{Q}_{p}(0):=\mathbb{Q}_{p}, \mathbb{Q}_{p}(1):=\left(\lim _{\mu^{n}}\right) \otimes_{\mathbb{Z}_{p}} \mathbb{Q}_{p}$. For $n \geqslant 1$, let $\mathbb{Q}_{p}(n):=\mathbb{Q}_{p}(1)^{\otimes n}$, $\mathbb{Q}_{p}(-n):=\operatorname{Hom}\left(\mathbb{Q}_{p}(n), \mathbb{Q}_{p}\right)$. Moreover, for a $\operatorname{Gal}\left(\bar{K}_{\mathfrak{p}} / K_{\mathfrak{p}}\right)$-representation $V$ over $\mathbb{Q}_{p}$, we define $V(n):=V \otimes_{\mathbb{Q}_{p}} \mathbb{Q}_{p}(n)$ on which $\operatorname{Gal}\left(\bar{K}_{\mathfrak{p}} / K_{\mathfrak{p}}\right)$ acts diagonally.

In $p$-adic Hodge theory the Hodge-Tate decomposition of $X$ is as follows:

$$
\bigoplus_{\substack{i, j \\ i+j=k}} H^{j}\left(X, \Omega_{X}^{i}\right) \otimes_{K} \mathbb{C}_{p}(-i) \cong H_{\text {ét }}^{k}\left(X_{\bar{K}_{\mathfrak{p}}}, \mathbb{Q}_{p}\right) \otimes_{\mathbb{Q}_{p}} \mathbb{C}_{p}
$$

This is an isomorphism of $\operatorname{Gal}\left(\bar{K}_{\mathfrak{p}} / K_{\mathfrak{p}}\right)$-representations, where $\operatorname{Gal}\left(\bar{K}_{\mathfrak{p}} / K_{\mathfrak{p}}\right)$ acts on $H^{i}\left(X, \Omega_{X}^{j}\right)$ trivially and on the right-hand side diagonally. This is a $p$-adic analogue of the usual Hodge decomposition over $\mathbb{C}$.

As a consequence, we can recover the Hodge numbers of $X$ from its $p$-adic Galois representations as follows:

$$
\operatorname{dim}_{K_{\mathfrak{p}}} H^{j}\left(X, \Omega_{X}^{i}\right)=\operatorname{dim}_{K_{\mathfrak{p}}}\left(H_{\text {ét }}^{i+j}\left(X_{\bar{K}_{\mathfrak{p}}}, \mathbb{Q}_{p}\right) \otimes_{\mathbb{Q}_{p}} \mathbb{C}_{p}(i)\right)^{\operatorname{Gal}\left(\bar{K}_{\mathfrak{p}} / K_{\mathfrak{p}}\right)},
$$

since $\left(\mathbb{C}_{p}\right)^{\operatorname{Gal}\left(\bar{K}_{\mathfrak{p}} / K_{\mathfrak{p}}\right)}=K_{\mathfrak{p}}$ and $\left(\mathbb{C}_{p}(i)\right)^{\operatorname{Gal}\left(\bar{K}_{\mathfrak{p}} / K_{\mathfrak{p}}\right)}=0$ for all $i \neq 0$ (see [Tat67, Theorem 2]).

Remark 4.1. A proof of Hodge-Tate decomposition was given by Faltings [Fal88] (for recent developments of Faltings' theory of almost étale extensions, also see [Fal02]). Tsuji gave another proof by reducing to the semistable reduction case by de Jong's alteration [Tsu99]. However, in this paper, we do not need the full version of the Hodge-Tate decomposition. For example, the result of Fontaine 


\section{T. Іто}

and Messing is enough for us [FM87]. They proved the Hodge-Tate decomposition when $K_{\mathfrak{p}}$ is unramified over $\mathbb{Q}_{p}, \operatorname{dim} X<p$, and $X$ has a proper smooth model over $\mathcal{O}_{K_{\mathfrak{p}}}$.

Remark 4.2. Moreover, by Lemma 4.3 below, we see that the semisimplification of the $p$-adic Galois representation determines the Hodge numbers by the same formula:

$$
\operatorname{dim}_{K_{\mathfrak{p}}} H^{j}\left(X, \Omega_{X}^{i}\right)=\operatorname{dim}_{K_{\mathfrak{p}}}\left(H_{\text {ét }}^{i+j}\left(X_{\bar{K}_{\mathfrak{p}}}, \mathbb{Q}_{p}\right)^{\mathrm{ss}} \otimes_{\mathbb{Q}_{p}} \mathbb{C}_{p}(i)\right)^{\operatorname{Gal}\left(\bar{K}_{\mathfrak{p}} / K_{\mathfrak{p}}\right)},
$$

where ss denotes the semisimplification as a $\operatorname{Gal}\left(\bar{K}_{\mathfrak{p}} / K_{\mathfrak{p}}\right)$-representation. This is a simple but important observation to consider the Hodge-Tate decomposition on the level of a Grothendieck group of Galois representations in $\S 5$.

Lemma 4.3 [It03, Lemma 4.4]. Let $0 \rightarrow V_{1} \rightarrow V_{2} \rightarrow V_{3} \rightarrow 0$ be an exact sequence of finitedimensional $\operatorname{Gal}\left(\bar{K}_{\mathfrak{p}} / K_{\mathfrak{p}}\right)$-representations over $\mathbb{Q}_{p}$. We define $h^{n}\left(V_{i}\right):=\operatorname{dim}\left(V_{i} \otimes_{\mathbb{Q}_{p}} \mathbb{C}_{p}(n)\right)^{\operatorname{Gal}\left(\bar{K}_{\mathfrak{p}} / K_{\mathfrak{p}}\right)}$ for $i=1,2,3$ and an integer $n$. Assume that $\operatorname{dim} V_{2}=\sum_{n} h^{n}\left(V_{2}\right)$. Then, we have $\operatorname{dim} V_{1}=$ $\sum_{n} h^{n}\left(V_{1}\right), \operatorname{dim} V_{3}=\sum_{n} h^{n}\left(V_{3}\right)$ and $h^{n}\left(V_{2}\right)=h^{n}\left(V_{1}\right)+h^{n}\left(V_{3}\right)$ for all $n$.

Proof. This lemma seems well known to specialists. However, we give the proof for the reader's convenience. In general, we have an inequality $\sum_{n} h^{n}\left(V_{i}\right) \leqslant \operatorname{dim} V_{i}$ for $i=1,3$ (for example, see [Fon94]). We shall prove these inequalities are in fact equalities. Since the functor $V \mapsto V^{\mathrm{Gal}\left(\bar{K}_{\mathfrak{p}} / K_{\mathfrak{p}}\right)}$ is left exact,

$$
\begin{aligned}
0 \longrightarrow & \left(V_{1} \otimes_{\mathbb{Q}_{p}} \mathbb{C}_{p}(n)\right)^{\operatorname{Gal}\left(\bar{K}_{\mathfrak{p}} / K_{\mathfrak{p}}\right)} \longrightarrow\left(V_{2} \otimes_{\mathbb{Q}_{p}} \mathbb{C}_{p}(n)\right)^{\operatorname{Gal}\left(\bar{K}_{\mathfrak{p}} / K_{\mathfrak{p}}\right)} \\
\longrightarrow & \left(V_{3} \otimes_{\mathbb{Q}_{p}} \mathbb{C}_{p}(n)\right)^{\operatorname{Gal}\left(\bar{K}_{\mathfrak{p}} / K_{\mathfrak{p}}\right)}
\end{aligned}
$$

is exact. Therefore $h^{n}\left(V_{2}\right) \leqslant h^{n}\left(V_{1}\right)+h^{n}\left(V_{3}\right)$ for all $n$. Then we have

$$
\begin{aligned}
\operatorname{dim} V_{2} & =\sum_{n} h^{n}\left(V_{2}\right) \leqslant \sum_{n} h^{n}\left(V_{1}\right)+\sum_{n} h^{n}\left(V_{3}\right) \leqslant \operatorname{dim} V_{1}+\operatorname{dim} V_{3} \\
& =\operatorname{dim} V_{2}
\end{aligned}
$$

and hence Lemma 4.3 is proved.

\section{Global Galois representations}

In this section, we recall some results on Galois representations over a number field.

\subsection{An application of the Chebotarev density theorem}

The following proposition is very important to work on the level of a Grothendieck group of Galois representations over a number field. This is an application of the Chebotarev density theorem in algebraic number theory.

Proposition 5.1 [Ser68, I.2.3]. Let $K$ be a number field and $l$ be a prime number. Let $V, V^{\prime}$ be two continuous l-adic Gal $(\bar{K} / K)$-representations such that they are unramified outside a finite set $S$ of maximal ideals of $\mathcal{O}_{K}$ and satisfy

$$
\operatorname{Tr}\left(\operatorname{Frob}_{\mathfrak{p}} ; V\right)=\operatorname{Tr}\left(\text { Frob }_{\mathfrak{p}} ; V^{\prime}\right) \text { for all } \mathfrak{p} \notin S .
$$

Then $V$ and $V^{\prime}$ have the same semisimplifications as $\operatorname{Gal}(\bar{K} / K)$-representations.

Proof. We only sketch the proof (for details, see [Ser68]). By the representation theory of a group over a field of characteristic 0 , the semisimplification of a $\mathrm{Gal}(\bar{K} / K)$-representation is determined by the traces of all elements in $\operatorname{Gal}(\bar{K} / K)$. Roughly speaking, the Chebotarev density theorem claims the set of conjugates of Frob $_{\mathfrak{p}}$ for $\mathfrak{p} \notin S$ is dense in $\operatorname{Gal}(\bar{K} / K)$. Since $V$ and $V^{\prime}$ are continuous 


\section{Stringy Hodge NUmbers}

representations, the equality of the traces of all Frob $\mathfrak{p}$ for $\mathfrak{p} \notin S$ implies the equality of traces of all elements in $\operatorname{Gal}(\bar{K} / K)$. Hence we have Proposition 5.1.

\subsection{Some Grothendieck groups of Galois representations}

Let $K$ be a number field. Let $S$ be a finite set of maximal ideals of $\mathcal{O}_{K}$. We fix a prime number $l=p$ and a maximal ideal $\mathfrak{p}$ dividing $p$. For every maximal ideal $\mathfrak{q}$ of $\mathcal{O}_{K}$, we fix an inclusion $\bar{K} \hookrightarrow \bar{K}_{\mathfrak{q}}$. Then we consider $\operatorname{Gal}\left(\bar{K}_{\mathfrak{q}} / K_{\mathfrak{q}}\right) \subset \operatorname{Gal}(\bar{K} / K)$ for all $\mathfrak{q}$.

Definition 5.2. Let $K(l, S, \mathfrak{p})$ be an abelian group generated by $\operatorname{Gal}(\bar{K} / K)$-representations $V$ satisfying the following conditions modulo an equivalence relation $\sim$ generated by $\left[V_{1}\right]+\left[V_{3}\right] \sim\left[V_{2}\right]$ for an exact sequence $0 \rightarrow V_{1} \rightarrow V_{2} \rightarrow V_{3} \rightarrow 0$ :

1) (unramifiedness outside $S$ ) For $\mathfrak{q} \notin S, I_{K_{\mathfrak{q}}}$ acts on $V$ trivially.

2) (weight filtration outside $S$ ) There exists a unique increasing $\operatorname{Gal}(\bar{K} / K)$-equivariant filtration $W$ on $V$ indexed by integers satisfying the following conditions:

a) $W_{k} V=0$ for $k \ll 0, W_{k} V=V$ for $k \gg 0$;

b) for every integer $k$ and $\mathfrak{q} \notin S$, the characteristic polynomial $P_{k}(t)=\operatorname{det}\left(1-t \cdot \operatorname{Frob}_{\mathfrak{q}} ; \mathrm{Gr}_{k}^{W} V\right)$ has integer coefficients and all complex absolute values of all conjugates of the roots of $P_{k}(t)$ are equal to $\left|\mathcal{O}_{K} / \mathfrak{q}\right|^{-k / 2}$.

$W$ is called the weight filtration on $V$.

3) (Hodge-Tate decomposition at $\mathfrak{p}$ ) For integers $i, j$, we define

$$
h_{\mathfrak{p}}^{i, j}(V):=\operatorname{dim}_{K_{\mathfrak{p}}}\left(\operatorname{Gr}_{i+j}^{W} V \otimes_{\mathbb{Q}_{p}} \mathbb{C}_{p}(i)\right)^{\operatorname{Gal}\left(\bar{K}_{\mathfrak{p}} / K_{\mathfrak{p}}\right)} .
$$

Then these numbers satisfy $\sum_{i, j} h_{\mathfrak{p}}^{i, j}(V)=\operatorname{dim}_{\mathbb{Q}_{p}} V$.

$K(l, S, \mathfrak{p})$ is called the Grothendieck group of $l$-adic $\operatorname{Gal}(\bar{K} / K)$-representations which are unramified outside $S$, have weight filtration, and have Hodge-Tate decomposition at $\mathfrak{p}$. Let $[V]$ denote the class of $V$ in $K(l, S, \mathfrak{p})$. An element in $K(l, S, \mathfrak{p})$ is called a virtual $\mathrm{Gal}(\bar{K} / K)$-representation.

Similarly, for an integer $k$, we define $K(l, S, \mathfrak{p}, k)$ as a subgroup of $K(l, S, \mathfrak{p})$ generated by $V$ satisfying $\operatorname{Gr}_{k}^{W} V=V$. The group $K(l, S, \mathfrak{p}, k)$ is called the Grothendieck group of $l$-adic $\operatorname{Gal}(\bar{K} / K)$ representations which are unramified outside $S$, have weight $k$, and have Hodge-Tate decomposition at $\mathfrak{p}$.

By the Jordan-Hölder theorem, $K(l, S, \mathfrak{p})$ is a free abelian group generated by simple $\mathrm{Gal}(\bar{K} / K)$ representations in $K(l, S, \mathfrak{p})$. Since $[V]=\sum_{k}\left[\operatorname{Gr}_{k}^{W} V\right]$ in $K(l, S, \mathfrak{p})$, a simple $\operatorname{Gal}(\bar{K} / K)$-representation has only one weight. Therefore we have a direct sum decomposition as follows:

$$
K(l, S, \mathfrak{p})=\bigoplus_{k \in \mathbb{Z}} K(l, S, \mathfrak{p}, k) .
$$

We define a ring structure on $K(l, S, \mathfrak{p})$ by extending the tensor product $\left[V_{1}\right] \cdot\left[V_{2}\right]=\left[V_{1} \otimes V_{2}\right]$. Then $K(l, S, \mathfrak{p})$ has a structure of a graded ring by the direct sum decomposition as above.

Definition 5.3. For a $\operatorname{Gal}(\bar{K} / K)$-representation $V$ in $K(l, S, \mathfrak{p})$, we define the $p$-adic E-function of $V$ as follows:

$$
E_{\mathfrak{p}}(V ; u, v):=\sum_{i, j} h_{\mathfrak{p}}^{i, j}(V) u^{i} v^{j}
$$

Remark 5.4. It is easy to see that the $p$-adic E-function satisfies the following properties (see Remark 4.2): 
1) for an exact sequence $0 \rightarrow V_{1} \rightarrow V_{2} \rightarrow V_{3} \rightarrow 0$, we have $E_{\mathfrak{p}}\left(V_{1} ; u, v\right)+E_{\mathfrak{p}}\left(V_{3} ; u, v\right)=E_{\mathfrak{p}}\left(V_{2} ; u, v\right)$;

2) for $V_{1}, V_{2}$, we have $E_{\mathfrak{p}}\left(V_{1} \otimes V_{2} ; u, v\right)=E_{\mathfrak{p}}\left(V_{1} ; u, v\right) \cdot E_{\mathfrak{p}}\left(V_{2} ; u, v\right)$.

Therefore, we can extend the $p$-adic $E$-function to a ring homomorphism

$$
E_{\mathfrak{p}}: K(l, S, \mathfrak{p}) \longrightarrow \mathbb{Z}[u, v] .
$$

\subsection{A variant: Galois representations with fractional weight filtration}

Let $d$ be an integer. Here we introduce a variant of $K(l, S, \mathfrak{p})$ whose weight filtration is indexed by elements of $(1 / d) \mathbb{Z}$ instead of $\mathbb{Z}$. This generalization is necessary to treat the $\mathbb{Q}$-Gorenstein case in the proof of Theorem 1.1.

Firstly, we introduce the fractional Tate twists $\mathbb{Q}_{p}(a)(a \in(1 / d) \mathbb{Z})$ as follows (for usual Tate twists, see $\S 4.3)$. Let $L$ be a field and $p$ be a prime number. The twist $\mathbb{Q}_{p}(1)$ is a one-dimensional $\operatorname{Gal}(\bar{L} / L)$-representation

$$
\rho: \operatorname{Gal}(\bar{L} / L) \longrightarrow \mathrm{GL}\left(\mathbb{Q}_{p}(1)\right) \cong \mathrm{GL}\left(1, \mathbb{Q}_{p}\right)=\mathbb{Q}_{p}^{\times}
$$

whose image is contained in $\mathbb{Z}_{p}^{\times}$. There exist open subgroups $U \subset \mathbb{Z}_{p}^{\times}, V \subset \mathbb{Z}_{p}$ on which $\log : U \rightarrow V$ and $\exp : V \rightarrow U$ converge. Therefore, if we replace $L$ by a finite extension of it,

$$
\rho_{1 / d}: \operatorname{Gal}(\bar{L} / L) \ni \sigma \mapsto \exp \left(\frac{1}{d} \log (\rho(\sigma))\right) \in \mathbb{Q}_{p}^{\times}
$$

is a one-dimensional $\operatorname{Gal}(\bar{L} / L)$-representation. We denote it by $\mathbb{Q}_{p}(1 / d)$. Then $\mathbb{Q}_{p}(1 / d)^{\otimes d} \cong \mathbb{Q}_{p}(1)$. For $n \in \mathbb{Z}, n \geqslant 1$, we define $\mathbb{Q}_{p}(n / d):=\mathbb{Q}_{p}(1 / d)^{\otimes n}, \mathbb{Q}_{p}(-n / d):=\operatorname{Hom}\left(\mathbb{Q}_{p}(n / d), \mathbb{Q}_{p}\right)$. If $L$ is a finite extension of $\mathbb{Q}_{p}$, we can similarly define $\mathbb{C}_{p}(a)(a \in(1 / d) \mathbb{Z})$ as in $\S 4.3$.

We define $K(l, S, \mathfrak{p})_{1 / d}$ as follows. Let the notation be the same as in $\S 5.2$. Then $K(l, S, \mathfrak{p})_{1 / d}$ is an abelian group generated by $\operatorname{Gal}(\bar{K} / K)$-representations $V$ satisfying the following conditions modulo an equivalence relation $\sim$ as in Definition 5.2.

1) The conditions 1 and 2 in Definition 5.2 hold, but we allow $k$ to be an element of $(1 / d) \mathbb{Z}$ instead of $\mathbb{Z}$.

2) Let $L$ be a finite extension of $K_{\mathfrak{p}}$ such that $\mathbb{Q}_{p}(1 / d)$ exists as a $\operatorname{Gal}(\bar{L} / L)$-representation. For $i, j \in(1 / d) \mathbb{Z}$, we define

$$
h_{\mathfrak{p}}^{i, j}(V):=\operatorname{dim}_{L}\left(\operatorname{Gr}_{i+j}^{W} V \otimes_{\mathbb{Q}_{p}} \mathbb{C}_{p}(i)\right)^{\operatorname{Gal}(\bar{L} / L)} .
$$

Then these numbers satisfy $\sum_{i, j \in(1 / d) \mathbb{Z}} h_{\mathfrak{p}}^{i, j}(V)=\operatorname{dim}_{\mathbb{Q}_{p}} V$. It is easy to see that this condition is independent of the choice of $L$.

Similarly, we can define $K(l, S, \mathfrak{p}, k)_{1 / d}$ as in $\S 5.2$. We have a direct sum decomposition as follows:

$$
K(l, S, \mathfrak{p})_{1 / d}=\bigoplus_{k \in(1 / d) \mathbb{Z}} K(l, S, \mathfrak{p}, k)_{1 / d}
$$

The group $K(l, S, \mathfrak{p})_{1 / d}$ has a ring structure. Moreover, we can define a $p$-adic $E$-function $E_{\mathfrak{p}}(V ; u, v)$ $\in \mathbb{Z}\left[u^{1 / d}, v^{1 / d}\right]$ for a $\operatorname{Gal}(\bar{K} / K)$-representation $V$ in $K(l, S, \mathfrak{p})_{1 / d}$. We can extend this to a ring homomorphism

$$
E_{\mathfrak{p}}: K(l, S, \mathfrak{p})_{1 / d} \longrightarrow \mathbb{Z}\left[u^{1 / d}, v^{1 / d}\right]
$$

Example 5.5. Assume that $\mathbb{Q}_{p}(1 / d)$ exists as a $\operatorname{Gal}(\bar{K} / K)$-representation. Note that this is satisfied if we replace $K$ by a finite extension of it. For $n \in \mathbb{Z}, \mathbb{Q}_{p}(n / d)$ is in $K(l, S, \mathfrak{p},-2 n / d)_{1 / d}$ such that $E_{\mathfrak{p}}\left(\mathbb{Q}_{p}(n / d) ; u, v\right)=u^{-n / d} v^{-n / d}$. 


\section{STRINGY HodGe NUMBERS}

\section{Conclusion: the number of $\mathbb{F}_{q}$-rational points, Galois representations and Hodge numbers}

We combine the results in $\S \S 4$ and 5 . Let the notation be the same as in $\S 5.2$.

Let $X$ be a proper smooth variety over $K$ which has a proper smooth model $\mathfrak{X}$ over $\left(\operatorname{Spec} \mathcal{O}_{K}\right) \backslash S$. Then $H_{\text {ét }}^{k}\left(X_{\bar{K}}, \mathbb{Q}_{l}\right)$ is a $\operatorname{Gal}(\bar{K} / K)$-representation in $K(l, S, \mathfrak{p}, k)$ by the Weil conjecture and $p$-adic Hodge theory (see $\S 4$ ). Let $X_{\bar{K}}=X \otimes_{K} \bar{K}$ and $X_{\mathbb{C}}=X \otimes_{K} \mathbb{C}$. We define a virtual representation

$$
\left[H_{\text {èt }}^{*}\left(X_{\bar{K}}, \mathbb{Q}_{l}\right)\right]:=\sum_{k}(-1)^{k}\left[H_{\text {ét }}^{k}\left(X_{\bar{K}}, \mathbb{Q}_{l}\right)\right]
$$

as an element in $K(l, S, \mathfrak{p})$. Then we have the equality of two $E$-functions

$$
E\left(X_{\mathbb{C}} ; u, v\right)=E_{\mathfrak{p}}\left(\left[H_{\text {ét }}^{*}\left(X_{\bar{K}}, \mathbb{Q}_{l}\right)\right] ; u, v\right)
$$

by comparing the Hodge decomposition of $X_{\mathbb{C}}$ and the Hodge-Tate decomposition of $X_{K_{\mathfrak{p}}}=$ $X \otimes_{K} K_{\mathfrak{p}}$.

\subsection{The proper smooth case}

By combining results in $\S \S 4$ and 5, we have the following results which connect the number of rational points and the Hodge numbers.

Proposition 6.1. Let $X$ be a proper smooth variety over $K$ which has a proper smooth model $\mathfrak{X}$ over $\left(\operatorname{Spec} \mathcal{O}_{K}\right) \backslash S$. Then we have

$$
\left|\mathfrak{X}\left(\mathcal{O}_{K} / \mathfrak{p}\right)\right|=\operatorname{Tr}\left(\operatorname{Frob}_{\mathfrak{p}} ;\left[H_{\text {ét }}^{*}\left(X_{\bar{K}}, \mathbb{Q}_{l}\right)\right]\right) \quad \text { for all } \mathfrak{p} \notin S .
$$

Proof. This follows from the Lefschetz trace formula for étale cohomology as in $\S 4.2$.

Corollary 6.2. Let $X$ (respectively $Y$ ) be a proper smooth variety over $K$ which has a proper smooth model $\mathfrak{X}$ (respectively $\mathfrak{Y})$ over $\left(\operatorname{Spec} \mathcal{O}_{K}\right) \backslash S$. If $\left|\mathfrak{X}\left(\mathcal{O}_{K} / \mathfrak{p}\right)\right|=\left|\mathfrak{Y}\left(\mathcal{O}_{K} / \mathfrak{p}\right)\right|$ for all $\mathfrak{p} \notin S$, then $\left[H_{\text {ét }}^{*}\left(X_{\bar{K}}, \mathbb{Q}_{l}\right)\right]=\left[H_{\text {ét }}^{*}\left(Y_{\bar{K}}, \mathbb{Q}_{l}\right)\right]$ in $K(l, S, \mathfrak{p})$. Therefore, we have

$$
E\left(X_{\mathbb{C}} ; u, v\right)=E_{\mathfrak{p}}\left(\left[H_{\text {ét }}^{*}\left(X_{\bar{K}}, \mathbb{Q}_{l}\right)\right] ; u, v\right)=E_{\mathfrak{p}}\left(\left[H_{\text {ét }}^{*}\left(Y_{\bar{K}}, \mathbb{Q}_{l}\right)\right] ; u, v\right)=E\left(Y_{\mathbb{C}} ; u, v\right) .
$$

Namely, the Hodge numbers of $X_{\mathbb{C}}$ and $Y_{\mathbb{C}}$ are equal.

Proof. By Proposition 6.1, we have

$$
\operatorname{Tr}\left(\operatorname{Frob}_{\mathfrak{p}} ;\left[H_{\text {ét }}^{*}\left(X_{\bar{K}}, \mathbb{Q}_{l}\right)\right]\right)=\operatorname{Tr}\left(\operatorname{Frob}_{\mathfrak{p}} ;\left[H_{\text {ét }}^{*}\left(Y_{\bar{K}}, \mathbb{Q}_{l}\right)\right]\right) \quad \text { for all } \mathfrak{p} \notin S .
$$

Hence we have $\left[H_{\text {ét }}^{*}\left(X_{\bar{K}}, \mathbb{Q}_{l}\right)\right]=\left[H_{\text {ét }}^{*}\left(Y_{\bar{K}}, \mathbb{Q}_{l}\right)\right]$ in $K(l, S, \mathfrak{p})$ by Proposition 5.1.

\subsection{A generalization: the open smooth case}

Next we generalize Proposition 6.1 to open smooth varieties by a method of Deligne in [Del71a, Del71b].

Let $X$ be a smooth variety over $K$ of dimension $n$ which is not necessarily proper. Assume that there exists a proper smooth variety $\bar{X} \supset X$ over $K$ such that $\bar{X} \backslash X=\bigcup_{i=1}^{r} D_{i}$ is a SNCD on $\bar{X}$. Let $I=\{1, \ldots, r\}, D_{J}=\bigcap_{j \in J} D_{j}$ for a nonempty subset $J \subset I$, and $D_{\emptyset}=\bar{X}$. We consider the following formal sum

$$
\left[H_{\mathrm{c}, \text { ét }}^{*}\left(X_{\bar{K}}, \mathbb{Q}_{l}\right)\right]:=\sum_{k}(-1)^{k}\left[H_{\mathrm{c}, \text { ét }}^{k}\left(X_{\bar{K}}, \mathbb{Q}_{l}\right)\right]
$$

where $H_{\mathrm{c} \text {,ét }}^{k}$ denotes étale cohomology with compact support. By the following Lemma 6.3, we see that the above is an equality in $K(l, S, \mathfrak{p})$. 
Lemma 6.3. Let $X$ be as above. Then we have

$$
\begin{aligned}
E\left(X_{\mathbb{C}} ; u, v\right) & =\sum_{J \subset I}(-1)^{|J|} E\left(\left(D_{J}\right)_{\mathbb{C}} ; u, v\right) \in \mathbb{Z}[u, v], \\
{\left[H_{\mathrm{c}, \text { ét }}^{*}\left(X_{\bar{K}}, \mathbb{Q}_{l}\right)\right] } & =\sum_{J \subset I}(-1)^{|J|}\left[H_{\text {ét }}^{*}\left(\left(D_{J}\right)_{\bar{K}}, \mathbb{Q}_{l}\right)\right] \in K(l, S, \mathfrak{p}) .
\end{aligned}
$$

Furthermore, we have the equality of two E-functions for $X$ :

$$
E\left(X_{\mathbb{C}} ; u, v\right)=E_{\mathfrak{p}}\left(\left[H_{\mathrm{c}, \text { ét }}^{*}\left(X_{\bar{K}}, \mathbb{Q}_{l}\right)\right] ; u, v\right) .
$$

Proof. Since $D_{J}$ is a proper smooth variety over $K$, we have

$$
E\left(\left(D_{J}\right)_{\mathbb{C}} ; u, v\right)=E_{\mathfrak{p}}\left(\left[H_{\text {ét }}^{*}\left(\left(D_{J}\right)_{\bar{K}}, \mathbb{Q}_{l}\right)\right] ; u, v\right) .
$$

Therefore, the second assertion immediately follows from the first assertion.

We only prove the first assertion for $E\left(X_{\mathbb{C}} ; u, v\right)$ since we can prove the case of $\left[H_{\mathrm{c}, \text { ét }}^{*}\left(X_{\bar{K}}, \mathbb{Q}_{l}\right)\right]$ in the same way. The Leray spectral sequence for the inclusion $X_{\mathbb{C}} \hookrightarrow \bar{X}_{\mathbb{C}}$ induces a spectral sequence

$$
E_{2}^{i, j}=\bigoplus_{\substack{J \subset I \\|J|=j}} H^{i}\left(\left(D_{J}\right)_{\mathbb{C}}, \mathbb{Q}\right)(-j) \Rightarrow H^{i+j}\left(X_{\mathbb{C}}, \mathbb{Q}\right),
$$

which defines the canonical mixed Hodge structure on $H^{k}\left(X_{\mathbb{C}}, \mathbb{Q}\right.$ ) (see [Del71a, (6.2)] and $[$ Del71b, $\S 3.2])$. For a finite-dimensional $\mathbb{Q}$-vector space $V$ with mixed Hodge structure, we define $E(V ; u, v)=\sum_{i, j} h^{i, j}\left(\mathrm{Gr}_{i+j}^{W} V\right) u^{i} v^{j}$. Note that

$$
E\left(X_{\mathbb{C}} ; u, v\right)=\sum_{k=1}^{2 n}(-1)^{k} E\left(H_{\mathrm{c}}^{k}\left(X_{\mathbb{C}}, \mathbb{Q}\right) ; u, v\right)
$$

by definition. By the above spectral sequence, we have

$$
\sum_{i, j}(-1)^{i+j} E\left(E_{2}^{i, j} ; u, v\right)=\sum_{k=1}^{2 n}(-1)^{k} E\left(H^{k}\left(X_{\mathbb{C}}, \mathbb{Q}\right) ; u, v\right) .
$$

By Poincaré duality, $H^{k}\left(X_{\mathbb{C}}, \mathbb{Q}\right)$ is dual to $H_{\mathrm{c}}^{2 n-k}\left(X_{\mathbb{C}}, \mathbb{Q}\right)(n)$. Then we have

$$
E\left(H^{k}\left(X_{\mathbb{C}}, \mathbb{Q}\right) ; u, v\right)=(u v)^{n} E\left(H_{\mathrm{c}}^{2 n-k}\left(X_{\mathbb{C}}, \mathbb{Q}\right) ; u^{-1}, v^{-1}\right) .
$$

On the other hand, since $D_{J}$ is a proper smooth variety of dimension $n-|J|, H^{i}\left(\left(D_{J}\right)_{\mathbb{C}}, \mathbb{Q}\right)(-|J|)$ is dual to $H^{2 n-2|J|-i}\left(\left(D_{J}\right)_{\mathbb{C}}, \mathbb{Q}\right)(n)$ by Poincaré duality. Hence we have

$$
E\left(E_{2}^{i, j} ; u, v\right)=\sum_{|J|=j}(u v)^{n} E\left(H^{2 n-2|J|-i}\left(\left(D_{J}\right)_{\mathbb{C}}, \mathbb{Q}\right) ; u^{-1}, v^{-1}\right) .
$$

By combining them, we have Lemma 6.3.

Next we consider the number of rational points valued in finite fields. For open smooth varieties, we have the following generalization of Proposition 6.1.

Proposition 6.4. Let $X$ be a smooth variety over $K$. Assume that there exist a proper smooth scheme $\overline{\mathfrak{X}}$ over $\left(\operatorname{Spec} \mathcal{O}_{K}\right) \backslash S$ and an open subscheme $\mathfrak{X} \subset \overline{\mathfrak{X}}$ whose generic fiber is $X$ such that $\overline{\mathfrak{X}} \backslash \mathfrak{X}=\bigcup_{i=1}^{r} \mathfrak{D}_{i}$ is a relative $S N C D$ on $\overline{\mathfrak{X}} /\left(\operatorname{Spec} \mathcal{O}_{K}\right) \backslash S$ (see Definition 3.3). Then we have

$$
\left|\mathfrak{X}\left(\mathcal{O}_{K} / \mathfrak{p}\right)\right|=\operatorname{Tr}\left(\operatorname{Frob}_{\mathfrak{p}} ;\left[H_{\text {c,ét }}^{*}\left(X_{\bar{K}}, \mathbb{Q}_{l}\right)\right]\right) \quad \text { for all } \mathfrak{p} \notin S .
$$




\section{StRingy Hodge NUMBers}

Proof. Let $I=\{1, \ldots, r\}, \mathfrak{D}_{J}=\bigcap_{j \in J} \mathfrak{D}_{j}$ for a nonempty subset $J \subset I$, and $\mathfrak{D}_{\emptyset}=\overline{\mathfrak{X}}$. Then, by the inclusion-exclusion principle, we have

$$
\left|\mathfrak{X}\left(\mathcal{O}_{K} / \mathfrak{p}\right)\right|=\sum_{J \subset I}(-1)^{|J|}\left|\mathfrak{D}_{J}\left(\mathcal{O}_{K} / \mathfrak{p}\right)\right| \quad \text { for all } \mathfrak{p} \notin S .
$$

Since $\mathfrak{D}_{J}$ is proper and smooth over $\left(\operatorname{Spec} \mathcal{O}_{K}\right) \backslash S$, we have

$$
\left|\mathfrak{D}_{J}\left(\mathcal{O}_{K} / \mathfrak{p}\right)\right|=\operatorname{Tr}\left(\text { Frob }_{\mathfrak{p}} ;\left[H_{\text {ét }}^{*}\left(\left(D_{J}\right)_{\bar{K}}, \mathbb{Q}_{l}\right)\right]\right)
$$

by Proposition 6.1. On the other hand, we have

$$
\operatorname{Tr}\left(\operatorname{Frob}_{\mathfrak{p}} ;\left[H_{\mathrm{c}, \text { ét }}^{*}\left(X_{\bar{K}}, \mathbb{Q}_{l}\right)\right]\right)=\sum_{J \subset I}(-1)^{|J|} \operatorname{Tr}\left(\operatorname{Frob}_{\mathfrak{p}} ;\left[H_{\text {ét }}^{*}\left(\left(D_{J}\right)_{\bar{K}}, \mathbb{Q}_{l}\right)\right]\right)
$$

by Lemma 6.3. By combining them, we have Proposition 6.4.

We note the following generalization of Corollary 6.2 for open smooth varieties, although we do not use it later.

Corollary 6.5. Let $X$ (respectively $Y$ ) be a smooth variety over $K$ satisfying the assumptions in Proposition 6.4. Let $\mathfrak{X} \subset \overline{\mathfrak{X}}$ (respectively $\mathfrak{Y} \subset \overline{\mathfrak{Y}}$ ) be a scheme over ( $\left.\operatorname{Spec} \mathcal{O}_{K}\right) \backslash S$ as in Proposition 6.4. If $\left|\mathfrak{X}\left(\mathcal{O}_{K} / \mathfrak{p}\right)\right|=\left|\mathfrak{Y}\left(\mathcal{O}_{K} / \mathfrak{p}\right)\right|$ for all $\mathfrak{p} \notin S$, then $\left[H_{\text {c,ét }}^{*}\left(X_{\bar{K}}, \mathbb{Q}_{l}\right)\right]=\left[H_{\text {c,ét }}^{*}\left(Y_{\bar{K}}, \mathbb{Q}_{l}\right)\right]$ in $K(l, S, \mathfrak{p})$. Therefore, we have

$$
E\left(X_{\mathbb{C}} ; u, v\right)=E_{\mathfrak{p}}\left(\left[H_{\mathrm{c}, \text { ét }}^{*}\left(X_{\bar{K}}, \mathbb{Q}_{l}\right)\right] ; u, v\right)=E_{\mathfrak{p}}\left(\left[H_{\mathrm{c}, \text { ét }}^{*}\left(Y_{\bar{K}}, \mathbb{Q}_{l}\right)\right] ; u, v\right)=E\left(Y_{\mathbb{C}} ; u, v\right) .
$$

Proof. The proof is the same as for Corollary 6.2. By Proposition 6.4, we have

$$
\operatorname{Tr}\left(\operatorname{Frob}_{\mathfrak{p}} ;\left[H_{\mathrm{c}, \text { ét }}^{*}\left(X_{\bar{K}}, \mathbb{Q}_{l}\right)\right]\right)=\operatorname{Tr}\left(\operatorname{Frob}_{\mathfrak{p}} ;\left[H_{\mathrm{c}, \text { ét }}^{*}\left(Y_{\bar{K}}, \mathbb{Q}_{l}\right)\right]\right) \quad \text { for all } \mathfrak{p} \notin S .
$$

Hence we have $\left[H_{\mathrm{c} \text {,ét }}^{*}\left(X_{\bar{K}}, \mathbb{Q}_{l}\right)\right]=\left[H_{\mathrm{c}, \text { ét }}^{*}\left(Y_{\bar{K}}, \mathbb{Q}_{l}\right)\right]$ in $K(l, S, \mathfrak{p})$ by Proposition 5.1 . The equality of the two $E$-functions follows from Lemma 6.3.

\section{Proof of the Main Theorem}

LEMma 7.1. Let $f: \mathfrak{X} \rightarrow T$ be a proper smooth morphism of schemes of characteristic 0 and $\mathfrak{D}=\bigcup_{i=1}^{r} \mathfrak{D}_{i}$ be a relative $S N C D$ on $\mathfrak{X} / T$ (see Definition 3.3). Assume that $T$ is connected. Then, all fibers of $\mathfrak{X} \backslash \mathfrak{D} \rightarrow T$ have the same E-functions as defined in Definition 2.1.

Proof. For a nonempty subset $J \subset\{1, \ldots, r\}, \mathfrak{D}_{J}=\bigcap_{j \in J} \mathfrak{D}_{j}$ is proper and smooth over $T$. Hence by the theorem of Deligne [Del68, 5.5], the Hodge numbers of all fibers of $\mathfrak{D}_{J} \rightarrow T$ are the same. On the other hand, the $E$-function of a fiber of $\mathfrak{X} \backslash \mathfrak{D} \rightarrow T$ can be computed from the Hodge numbers of a fiber of $\mathfrak{D}_{J} \rightarrow T$ by Lemma 6.3. Therefore, we have Lemma 7.1.

Proof of Theorem 1.1. Let $\rho: Y \rightarrow X$ and $\rho^{\prime}: Y^{\prime} \rightarrow X$ be as in Theorem 1.1. Let $n$ be the dimension of $X$. To avoid confusion, here $E_{\mathrm{st}}(X ; u, v)_{\rho}$ (respectively $\left.E_{\mathrm{st}}(X ; u, v)_{\rho^{\prime}}\right)$ denotes the stringy $E$-function of $X$ defined by $\rho: Y \rightarrow X$ (respectively $\rho^{\prime}: Y^{\prime} \rightarrow X$ ) as in Definition 2.4. We shall prove the equality $E_{\mathrm{st}}(X ; u, v)_{\rho}=E_{\mathrm{st}}(X ; u, v)_{\rho^{\prime}}$.

Step 1. Let $f: Y \rightarrow Y^{\prime}$ be a birational map between $Y$ and $Y^{\prime}$ over $X$. Let $Z$ be a resolution of singularities of the closure of the graph of $f$ such that the exceptional divisor of $Z \rightarrow X$ is a SNCD. Let $\tau: Z \rightarrow Y$ and $\tau^{\prime}: Z \rightarrow Y^{\prime}$ be natural morphisms. Then we have the following 
commutative diagram.

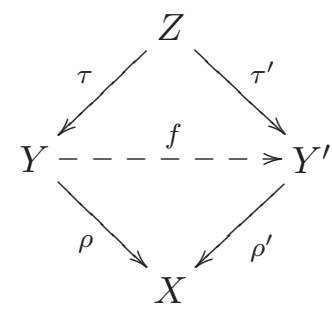

If we prove $E_{\mathrm{st}}(X ; u, v)_{\rho}=E_{\mathrm{st}}(X ; u, v)_{\rho \circ \tau}$ and $E_{\mathrm{st}}(X ; u, v)_{\rho^{\prime}}=E_{\mathrm{st}}(X ; u, v)_{\rho^{\prime} \circ \tau^{\prime}}$, then we have Theorem 1.1 since $\rho \circ \tau=\rho^{\prime} \circ \tau^{\prime}$. In the following, we only prove $E_{\mathrm{st}}(X ; u, v)_{\rho}=E_{\mathrm{st}}(X ; u, v)_{\rho \circ \tau}$ since we can similarly prove $E_{\mathrm{st}}(X ; u, v)_{\rho^{\prime}}=E_{\mathrm{st}}(X ; u, v)_{\rho^{\prime} \circ \tau^{\prime}}$.

Step 2. We shall show that we may assume everything is defined over a number field. Since $X, Y, Z, \rho, \tau$ are defined over a subfield $K^{\prime}$ of $\mathbb{C}$ which is finitely generated over $\mathbb{Q}$, there exists an irreducible variety $T$ over a number field $K$ such that the function field of $T$ is $K^{\prime}$. Furthermore, there exists a proper scheme $\widetilde{X}$, proper smooth schemes $\widetilde{Y}, \widetilde{Z}$, and proper birational morphisms $\tilde{\rho}: \widetilde{Y} \rightarrow \widetilde{X}, \tilde{\tau}: \widetilde{Z} \rightarrow \widetilde{Y}$ over $T$ such that $\widetilde{X} \times_{T} \operatorname{Spec} \mathbb{C}=X, \widetilde{Y} \times_{T} \operatorname{Spec} \mathbb{C}=Y, \widetilde{Z} \times_{T} \operatorname{Spec} \mathbb{C}=Z$, $\tilde{\rho} \times_{T} \operatorname{Spec} \mathbb{C}=\rho, \tilde{\tau} \times_{T} \operatorname{Spec} \mathbb{C}=\tau$. We write

$$
K_{Y}=\rho^{*} K_{X}+\sum_{i=1}^{r} a_{i} D_{i}, \quad K_{Z}=(\rho \circ \tau)^{*} K_{X}+\sum_{j=1}^{s} b_{j} E_{j},
$$

with $a_{i} \in \mathbb{Q}, a_{i}>-1, b_{j} \in \mathbb{Q}, b_{j}>-1$ (see Definition 2.3). Let $d$ be a positive integer such that $\left(K_{X}\right)^{\otimes d}$ is a Cartier divisor on $X$. Then $a_{i}, b_{j}$ are elements of $(1 / d) \mathbb{Z}$.

If we replace $T$ by a Zariski open subset of it, $\left(K_{X}\right)^{\otimes d}$ extends to a Cartier divisor $\left(\Omega_{\widetilde{X} / T}^{n}\right)^{\otimes d}$ on $\widetilde{X}$ over $T$, and we can write

$$
\Omega_{\widetilde{Y} / T}^{n}=\tilde{\rho}^{*} \Omega_{\widetilde{X} / T}^{n}+\sum_{i=1}^{r} a_{i} \widetilde{D}_{i}, \quad \Omega_{\widetilde{Z} / T}^{n}=(\tilde{\rho} \circ \tilde{\tau})^{*} \Omega_{\widetilde{X} / T}^{n}+\sum_{j=1}^{s} b_{j} \widetilde{E}_{j},
$$

where $\widetilde{D}=\bigcup_{i=1}^{r} \widetilde{D}_{i}$ (respectively $\widetilde{E}=\bigcup_{j=1}^{s} \widetilde{E}_{j}$ ) is a relative SNCD on $\widetilde{Y} / T$ (respectively $\widetilde{Z} / T$ ) (see Definition 3.3). If we replace $K$ by a finite extension of it, there exists a $K$-rational point $t \in T(K)$. Let $\widetilde{X}_{t}, \widetilde{Y}_{t}, \widetilde{Z}_{t}, \tilde{\rho}_{t}, \tilde{\tau}_{t}$ be the fibers at $t$. Then $E_{\mathrm{st}}(X ; u, v)_{\rho}=E_{\mathrm{st}}\left(\widetilde{X}_{t} ; u, v\right)_{\tilde{\rho}_{t}}$ and $E_{\mathrm{st}}(X ; u, v)_{\rho \circ \tau}=$ $E_{\mathrm{st}}\left(\widetilde{X}_{t} ; u, v\right)_{\tilde{\rho}_{t} \circ \tilde{\tau}_{t}}$ by Lemma 7.1. By replacing $X, Y, Z, \rho, \tau$ by $\widetilde{X}_{t}, \widetilde{Y}_{t}, \widetilde{Z}_{t}, \tilde{\rho}_{t}, \tilde{\tau}_{t}$, we may assume $X, Y, Z, \rho, \tau$ are defined over a number field $K$.

Step 3. By the same argument as above, there exists a finite set $S$ of maximal ideals of $\mathcal{O}_{K}$, a proper scheme $\mathfrak{X}$, proper smooth schemes $\mathfrak{Y}, \mathfrak{Z}$, and proper birational morphisms $\bar{\rho}: \mathfrak{Y} \rightarrow \mathfrak{X}$, $\bar{\tau}: \mathfrak{Z} \rightarrow \mathfrak{Y}$ over $\mathfrak{T}=\left(\operatorname{Spec} \mathcal{O}_{K}\right) \backslash S$ such that generic fibers of $\mathfrak{X}, \mathfrak{Y}, \mathfrak{Z}, \bar{\rho}, \bar{\tau}$ are $X, Y, Z, \rho, \tau$. By enlarging $S,\left(K_{X}\right)^{\otimes d}$ extends to a Cartier divisor $\left(\Omega_{\mathfrak{X} / \mathfrak{T}}^{n}\right)^{\otimes d}$ on $\mathfrak{X}$ over $\mathfrak{T}$ and we can write

$$
\Omega_{\mathfrak{Y} / \mathfrak{T}}^{n}=\bar{\rho}^{*} \Omega_{\mathfrak{X} / \mathfrak{T}}^{n}+\sum_{i=1}^{r} a_{i} \mathfrak{D}_{i}, \quad \Omega_{\mathfrak{Z} / \mathfrak{T}}^{n}=(\bar{\rho} \circ \bar{\tau})^{*} \Omega_{\mathfrak{X} / \mathfrak{T}}^{n}+\sum_{j=1}^{s} b_{j} \mathfrak{E}_{j},
$$

where $\mathfrak{D}=\bigcup_{i=1}^{r} \mathfrak{D}_{i}\left(\right.$ respectively $\left.\mathfrak{E}=\bigcup_{j=1}^{s} \mathfrak{E}_{j}\right)$ is a relative SNCD on $\mathfrak{Y} / \mathfrak{T}$ (respectively $\left.\mathfrak{Z} / \mathfrak{T}\right)$.

Step 4. Here we compute $p$-adic integration. Take a maximal ideal $\mathfrak{q} \notin S$. Let $K_{\mathfrak{q}}$ be a $\mathfrak{q}$-adic completion of $K$ and $\mathcal{O}_{K_{\mathfrak{q}}}$ be the ring of integers of $K_{\mathfrak{q}}$. Let $q=\left|\mathcal{O}_{K} / \mathfrak{q}\right|$ be the number of elements of the residue field. 


\section{STRINGy Hodge NUMBERS}

Let $\mathfrak{U}_{1}, \ldots, \mathfrak{U}_{k}$ be a finite open covering of $\mathfrak{X}$ over $\mathfrak{T}$ such that $\left(\Omega_{\mathfrak{X} / \mathfrak{T}}^{n}\right)^{\otimes d}$ is a trivial line bundle on each $\mathfrak{U}_{i}(1 \leqslant i \leqslant k)$. Let $\omega_{i}$ be a nowhere vanishing section of $\left(\Omega_{\mathfrak{X} / \mathfrak{T}}^{n}\right)^{\otimes d}$ on $\mathfrak{U}_{i}$.

Then, by Proposition 3.4, we can compute the $p$-adic integration of $\bar{\rho}^{*} \omega_{i}$ on $\bar{\rho}^{-1} \mathfrak{U}_{i}\left(\mathcal{O}_{K_{\mathfrak{q}}}\right)$ as follows:

$$
\int_{\bar{\rho}^{-1} \mathfrak{U}_{i}\left(\mathcal{O}_{K_{\mathfrak{q}}}\right)}\left|\bar{\rho}^{*} \omega_{i}\right|_{p}^{1 / d}=\frac{1}{q^{n}} \sum_{J \subset\{1, \ldots, r\}}\left|\left(\mathfrak{D}_{J}^{\circ} \cap \bar{\rho}^{-1} \mathfrak{U}_{i}\right)\left(\mathbb{F}_{q}\right)\right| \prod_{j \in J} \frac{q-1}{q^{a_{j}+1}-1},
$$

where $\mathfrak{D}_{J}^{\circ}$ are the same as in Proposition 3.4. Note that $\operatorname{div}\left(\bar{\rho}^{*} \omega_{i}\right)=\sum_{i=1}^{r} d a_{i} \mathfrak{D}_{i}$ since $\omega_{i}$ is a nowhere vanishing section of $\left(\Omega_{\mathfrak{X} / \mathfrak{T}}^{n}\right)^{\otimes d}$.

Similarly, for $(\bar{\rho} \circ \bar{\tau})^{*} \omega_{i}$, we have

$$
\int_{(\bar{\rho} \circ \bar{\tau})^{-1} \mathfrak{U}_{i}\left(\mathcal{O}_{K_{\mathfrak{q}}}\right)}\left|(\bar{\rho} \circ \bar{\tau})^{*} \omega_{i}\right|_{p}^{1 / d}=\frac{1}{q^{n}} \sum_{J^{\prime} \subset\{1, \ldots, s\}}\left|\left(\mathfrak{E}_{J^{\prime}}^{\circ} \cap(\bar{\rho} \circ \bar{\tau})^{-1} \mathfrak{U}_{i}\right)\left(\mathbb{F}_{q}\right)\right| \prod_{j^{\prime} \in J^{\prime}} \frac{q-1}{q^{b_{j^{\prime}}+1}-1},
$$

where $\mathfrak{E}_{J^{\prime}}^{\circ}$ is the same as above.

On the other hand, by the change-of-variable formula for $p$-adic integration, we have

$$
\int_{\bar{\rho}-1 \mathfrak{U}_{i}\left(\mathcal{O}_{K_{\mathfrak{q}}}\right)}\left|\bar{\rho}^{*} \omega_{i}\right|_{p}^{1 / d}=\int_{(\bar{\rho} \circ \bar{\tau})^{-1} \mathfrak{U}_{i}\left(\mathcal{O}_{K_{\mathfrak{q}}}\right)}\left|(\bar{\rho} \circ \bar{\tau})^{*} \omega_{i}\right|_{p}^{1 / d} .
$$

Since the same is true for all finite intersections of $\mathfrak{U}_{i}$, by the inclusion-exclusion principle, we conclude that

$$
\frac{1}{q^{n}} \sum_{J \subset\{1, \ldots, r\}}\left|\mathfrak{D}_{J}^{\circ}\left(\mathbb{F}_{q}\right)\right| \prod_{j \in J} \frac{q-1}{q^{a_{j}+1}-1}=\frac{1}{q^{n}} \sum_{J^{\prime} \subset\{1, \ldots, s\}}\left|\mathfrak{E}_{J^{\prime}}^{\circ}\left(\mathbb{F}_{q}\right)\right| \prod_{j^{\prime} \in J^{\prime}} \frac{q-1}{q^{b_{j^{\prime}}+1}-1} .
$$

Note that the above argument works for every $\mathfrak{q} \notin S$.

Step 5. Fix a prime number $l=p$ and a maximal ideal $\mathfrak{p}$ of $\mathcal{O}_{K}$ dividing $p$. By enlarging $S$, we may assume that $S$ contains all maximal ideals of $\mathcal{O}_{K}$ dividing $p$. We shall work on the level of the Grothendieck group $K(l, S, \mathfrak{p})_{1 / d}$ of $\operatorname{Gal}(\bar{K} / K)$-representations introduced in $\S 5$.

We rewrite the conclusion of Step 4 in the following form by multiplying with

$$
q^{n} \cdot \prod_{j=1}^{r}\left(q^{a_{j}+1}-1\right) \cdot \prod_{j^{\prime}=1}^{s}\left(q^{b_{j^{\prime}}+1}-1\right)
$$

on both sides:

$$
\begin{aligned}
& \prod_{j^{\prime}=1}^{s}\left(q^{b_{j^{\prime}}+1}-1\right) \sum_{J \subset\{1, \ldots, r\}}\left(\left|\mathfrak{D}_{J}^{\circ}\left(\mathbb{F}_{q}\right)\right| \prod_{j \in J}(q-1) \prod_{j \notin J}\left(q^{a_{j}+1}-1\right)\right) \\
& =\prod_{j=1}^{r}\left(q^{a_{j}+1}-1\right) \sum_{J^{\prime} \subset\{1, \ldots, s\}}\left(\left|\mathfrak{E}_{J^{\prime}}^{\circ}\left(\mathbb{F}_{q}\right)\right| \prod_{j^{\prime} \in J^{\prime}}(q-1) \prod_{j^{\prime} \notin J^{\prime}}\left(q^{b_{j^{\prime}}+1}-1\right)\right) .
\end{aligned}
$$

By replacing $K$ by a finite extension of it, we may assume that $\mathbb{Q}_{l}(1 / d)$ exists as a $\operatorname{Gal}(\bar{K} / K)$ representation (see Example 5.5). Recall that the image of $\operatorname{Frob}_{\mathfrak{q}}$ in $\mathrm{Gal}\left(\overline{\mathbb{F}}_{q} / \mathbb{F}_{q}\right)$ is the inverse of the $q$ th power automorphism $x \mapsto x^{q}$ of $\overline{\mathbb{F}}_{q}$. Therefore, for $m \in(1 / d) \mathbb{Z}, \operatorname{Tr}\left(\operatorname{Frob}_{\mathfrak{q}} ; \mathbb{Q}_{l}(m)\right)=q^{-m}$. 
Hence, we have the following equality in $K(l, S, \mathfrak{p})_{1 / d}$ :

$$
\begin{aligned}
& \prod_{j^{\prime}=1}^{s}\left(\left[\mathbb{Q}_{l}\left(-b_{j^{\prime}}-1\right)\right]-1\right) \sum_{J \subset\{1, \ldots, r\}}\left(\left[H_{\mathrm{c}, \text { ét }}^{*}\left(\left(D_{J}^{\circ}\right)_{\bar{K}}, \mathbb{Q}_{l}\right)\right] \prod_{j \in J}\left(\left[\mathbb{Q}_{l}(-1)\right]-1\right) \prod_{j \notin J}\left(\left[\mathbb{Q}_{l}\left(-a_{j}-1\right)\right]-1\right)\right) \\
& =\prod_{j=1}^{r}\left(\left[\mathbb{Q}_{l}\left(-a_{j}-1\right)\right]-1\right) \sum_{J^{\prime} \subset\{1, \ldots, s\}}\left(\left[H_{\mathrm{c}, \text { ét }}^{*}\left(\left(E_{J^{\prime}}^{\circ}\right)_{\bar{K}}, \mathbb{Q}_{l}\right)\right] \prod_{j^{\prime} \in J^{\prime}}\left(\left[\mathbb{Q}_{l}(-1)\right]-1\right) \prod_{j^{\prime} \notin J^{\prime}}\left(\left[\mathbb{Q}_{l}\left(-b_{j^{\prime}}-1\right)\right]-1\right)\right),
\end{aligned}
$$

since the traces of Frob $_{\mathfrak{q}}$ on both sides are equal for all $\mathfrak{q} \notin S$ (see Propositions 6.4 and 5.1). Note that $1 \in K(l, S, \mathfrak{p})_{1 / d}$ denotes the class of the trivial $\mathrm{Gal}(\bar{K} / K)$-representation.

Since $E_{\mathfrak{p}}\left(\mathbb{Q}_{l}(m) ; u, v\right)=u^{-m} v^{-m}$ for $m \in(1 / d) \mathbb{Z}$ by Example 5.5, we have

$$
\begin{aligned}
& \prod_{j^{\prime}=1}^{s}\left((u v)^{b_{j^{\prime}}+1}-1\right) \sum_{J \subset\{1, \ldots, r\}}\left(E\left(\left(D_{J}^{\circ}\right)_{\mathbb{C}} ; u, v\right) \prod_{j \in J}(u v-1) \prod_{j \notin J}\left((u v)^{a_{j}+1}-1\right)\right) \\
& =\prod_{j=1}^{r}\left((u v)^{a_{j}+1}-1\right) \sum_{J^{\prime} \subset\{1, \ldots, s\}}\left(E\left(\left(E_{J^{\prime}}^{\circ}\right)_{\mathbb{C}} ; u, v\right) \prod_{j^{\prime} \in J^{\prime}}(u v-1) \prod_{j^{\prime} \notin J^{\prime}}\left((u v)^{b_{j^{\prime}}+1}-1\right)\right)
\end{aligned}
$$

by Lemma 6.3. By Definition 2.4, this proves $E_{\mathrm{st}}\left(X_{\mathbb{C}} ; u, v\right)_{\rho}=E_{\mathrm{st}}\left(X_{\mathbb{C}} ; u, v\right)_{\rho \circ \tau}$ and hence Theorem 1.1.

Remark 7.2. If we take an appropriate $\mathfrak{p}$ in Step 5, we can use the result of Fontaine and Messing [FM87] (see Remark 4.1). Therefore, we do not need the full version of the Hodge-Tate decomposition for Theorem 1.1.

\section{ACKNOWLEDGEMENTS}

The author is grateful to Takeshi Saito and Kazuya Kato for their advice and support. He would like to thank Victor V. Batyrev and François Loeser for helpful discussions on motivic integration, Shinichi Mochizuki and Takeshi Tsuji for invaluable suggestions on $p$-adic Hodge theory, and Yujiro Kawamata, Daisuke Matsushita, Yasunari Nagai, Keiji Oguiso, Atsushi Takahashi, Hokuto Uehara, Ezra Getzler, Willem Veys and Chin-Lung Wang for comments and encouragement. He would also like to thank Yoichi Mieda for reading an earlier version carefully. The author was supported by the Japan Society for the Promotion of Science Research Fellowships for Young Scientists.

\section{REFERENCES}

AKMW02 D. Abramovich, K. Karu, K. Matsuki and J. Wlodarczyk, Torification and factorization of birational maps, J. Amer. Math. Soc. 15 (2002), 531-572.

Bat98 V. V. Batyrev, Stringy Hodge numbers of varieties with Gorenstein canonical singularities, in Integrable systems and algebraic geometry (Kobe/Kyoto, 1997) (World Scientific, River Edge, NJ, 1998), 1-32.

Bat99 V. V. Batyrev, Birational Calabi-Yau n-folds have equal Betti numbers, in New trends in algebraic geometry (Warwick, 1996) (Cambridge University Press, Cambridge, 1999), 1-11.

BB96 V. V. Batyrev and L. A. Borisov, Mirror duality and string-theoretic Hodge numbers, Invent. Math. 126 (1996), 183-203.

BD96 V. V. Batyrev and D. I. Dais, Strong McKay correspondence, string-theoretic Hodge numbers and mirror symmetry, Topology 35 (1996), 901-929.

BM01 L. A. Borisov and A. R. Mavlyutov, String cohomology of Calabi-Yau hypersurfaces via mirror symmetry, Adv. Math. 180 (2003), 355-390.

Del68 P. Deligne, Théorème de Lefschetz et critères de dégénérescence de suites spectrales, Publ. Math. Inst. Hautes Études Sci. 35 (1968), 259-278. 


\section{STRINGY HodGe NUMBERS}

Del71a P. Deligne, Théorie de Hodge I, in Actes du Congrès International des Mathématiciens (Nice, 1970), tome 1 (Gauthier-Villars, Paris, 1971), 425-430.

Del71b P. Deligne, Théorie de Hodge II, Publ. Math. Inst. Hautes Études Sci. 40 (1971), 5-57.

Del74a P. Deligne, La conjecture de Weil I, Publ. Math. Inst. Hautes Études Sci. 43 (1974), 273-307.

Del74b P. Deligne, Théorie de Hodge III, Publ. Math. Inst. Hautes Études Sci. 44 (1974), 5-77.

Del80 P. Deligne, La conjecture de Weil II, Publ. Math. Inst. Hautes Études Sci. 52 (1980), 137-252.

DL98 J. Denef and F. Loeser, Motivic Igusa zeta functions, J. Algebraic Geom. 7 (1998), 505-537.

DL99 J. Denef and F. Loeser, Germs of arcs on singular algebraic varieties and motivic integration, Invent. Math. 135 (1999), 201-232.

DL01 J. Denef and F. Loeser, Definable sets, motives and p-adic integrals, J. Amer. Math. Soc. 14 (2001), 429-469.

Fal88 G. Faltings, p-adic Hodge theory, J. Amer. Math. Soc. 1 (1988), 255-299.

Fal02 G. Faltings, Almost étale extensions. Cohomologies p-adiques et applications arithmétiques, II, Astérisque 279 (2002), 185-270.

FM87 J.-M. Fontaine and W. Messing, p-adic periods and p-adic étale cohomology, in Current trends in arithmetical algebraic geometry (Arcata, CA, 1985), Contemporary Mathematics, vol. 67 (American Mathematical Society, Providence, RI, 1987), 179-207.

Fon94 J.-M. Fontaine, Le corps des périodes p-adiques, with an appendix by Pierre Colmez, Périodes p-adiques (Bures-sur-Yvette, 1988), Astérisque 223 (1994), 59-111.

It01 T. Ito, A note on Hodge numbers of Calabi-Yau manifolds, Master's thesis, University of Tokyo (2001).

It03 T. Ito, Birational smooth minimal models have equal Hodge numbers in all dimensions, in Calabi-Yau varieties and mirror symmetry (Toronto, ON, 2001), Fields Inst. Commun. 38 (American Mathematical Society, Providence, RI, 2003), 183-194.

Kaw88 Y. Kawamata, Crepant blowing-up of 3-dimensional canonical singularities and its application to degenerations of surfaces, Ann. of Math. (2) 127 (1988), 93-163.

Kol89 J. Kollár, Flops, Nagoya Math. J. 113 (1989), 15-36.

KMM85 Y. Kawamata, K. Matsuda and K. Matsuki, Introduction to the minimal model problem, in Algebraic geometry, Sendai, 1985, Advanced Studies in Pure Mathematics, vol. 10 (NorthHolland, Amsterdam, 1985), 283-360.

Mor99 D. R. Morrison, The geometry underlying mirror symmetry, in New trends in algebraic geometry (Warwick, 1996) (Cambridge University Press, Cambridge, 1999), 283-310.

Ser68 J.-P. Serre, Abelian l-adic representations and elliptic curves (W. A. Benjamin, New York, 1968).

Tat67 J. T. Tate, p-divisible groups, in Proc. conf. local fields (Driebergen, 1966) (Springer, Berlin, 1967), 158-183.

Tsu99 T. Tsuji, p-adic étale cohomology and crystalline cohomology in the semi-stable reduction case, Invent. Math. 137 (1999), 233-411.

Vey01 W. Veys, Zeta functions and 'Kontsevich invariants' on singular varieties, Canad. J. Math. 53 (2001), 834-865.

Vey03 W. Veys, Stringy zeta functions for $\mathbb{Q}$-Gorenstein varieties, Duke Math. J. 120 (2003), 469-514.

Wan98 C.-L. Wang, On the topology of birational minimal models, J. Differential Geom. 50 (1998), 129-146.

Wan2a C.-L. Wang, Cohomology theory in birational geometry, J. Differential Geom. 60 (2002), 345-354.

Wan2b C.-L. Wang, K-equivalence in birational geometry, Preprint (2002), math.AG/0204160.

Wei82 A. Weil, Adeles and algebraic groups (Birkhäuser, Boston, 1982).

Tetsushi Ito tetsushi@math.kyoto-u.ac.jp

Department of Mathematics, Faculty of Science, Kyoto University, Kyoto 606-8502, Japan 